\title{
ARL ACADEMIC LAW LibraRY STATISTICS 2006-2007
}

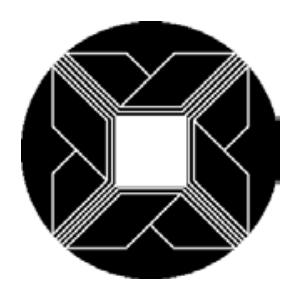

Compiled and Edited by

Martha Kyrillidou

Les Bland

Association of Research Libraries

WASHINGTON, DC

2008 
ARL Academic Law Library Statistics 2006-2007

The tables presented in this publication are not indicative of performance and outcomes and should not be used as measures of library quality. In comparing any individual library to ARL medians or to other ARL members, one must be careful to make such comparisons within the context of differing institutional and local goals and characteristics.

The ARL Academic Law Library Statistics datafiles and accompanying documentation are available at www.arl.org/stats/annualsurveys/law/index.shtml.

Published by the

Association of Research Libraries

Washington, DC 20036

www.arl.org

ISSN 1538-8999

ISBN 1-59407-811-4

EAN 978-159407-811-8

(C) 2008

The compilation is copyrighted by the Association of Research Libraries. Blanket permission is granted to reproduce and distribute copies of this work for nonprofit, educational, or library purposes, provided that the author, source, and copyright notice are included on each copy. This permission is in addition to rights of reproduction granted under Sections 107, 108, and other provisions of the US Copyright Act.

The paper used in this publication meets the minimum requirements of the American National Standard for Information Science and National Information Standards Organization standard-Permanence of Paper for Publications and Documents in Libraries and Archives, ANSI/NISO Z39.48-1992(R1997). 


\section{CONTENTS}

Hightlights: ARL Academic Law Library Statistics 2006-2007

Data Tables 2006-2007

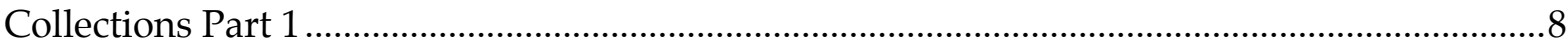

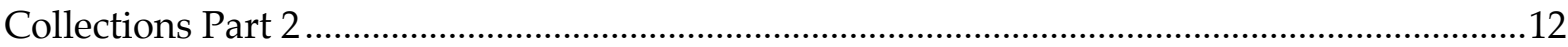

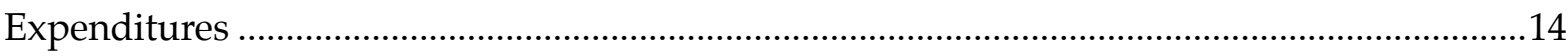

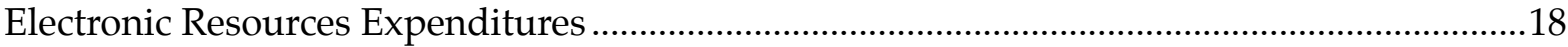

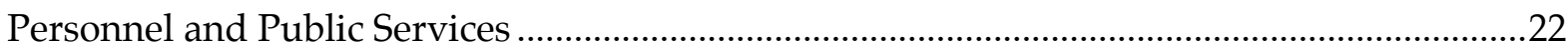

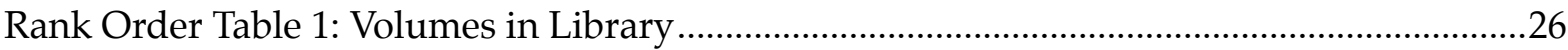

Rank Order Table 2: Volumes Added (Gross) ..........................................................................27

Rank Order Table 3: Current Serials (Total) .....................................................................28

Rank Order Table 4: Total Library Expenditures .................................................................29

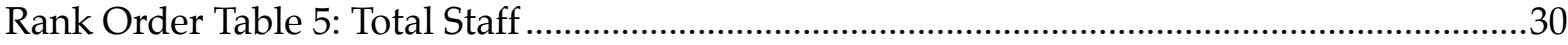

Rank Order Table 6: Total Electronic Resources.................................................................31

ARL Statistics Questionnaire 2006-2007: Instructions for Completing the Questionnaire..........33

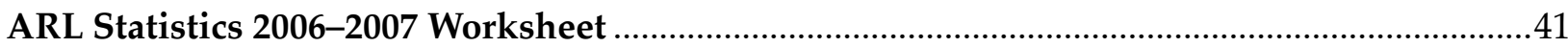

Footnotes to the ARL Academic Law Library Statistics 2006-2007 ........................................47

Appendix: ARL Member Libraries as of June 1, 2008 ….....................................................53 



\section{Highlights}

\section{ARL Academic Law Library Statistics 2006-2007}

- Out of 113 ARL university libraries, 74 responded to this survey.

- Law libraries reported median values of 332,247 volumes held and 8,399 gross volumes added. Also, these libraries employed the full-time equivalent of 2,201 staff members in the fiscal year 2006-2007.

- Responding libraries reported total expenditures of $\$ 208,820,262 .^{1}$ As seen in the graph below, materials expenditures made up the largest portion of the total, with $\%$ of aggregated expenses falling under a materials-related category.

- Law libraries reported a total of $\$ 17,259,064$ on electronic resources, or a median of $18.52 \%$ of their total materials budgets. This includes a total of $\$ 14,864,912$ on electronic serials.

\section{Expenditures in ARL Academic Law Libraries 2006-2007}

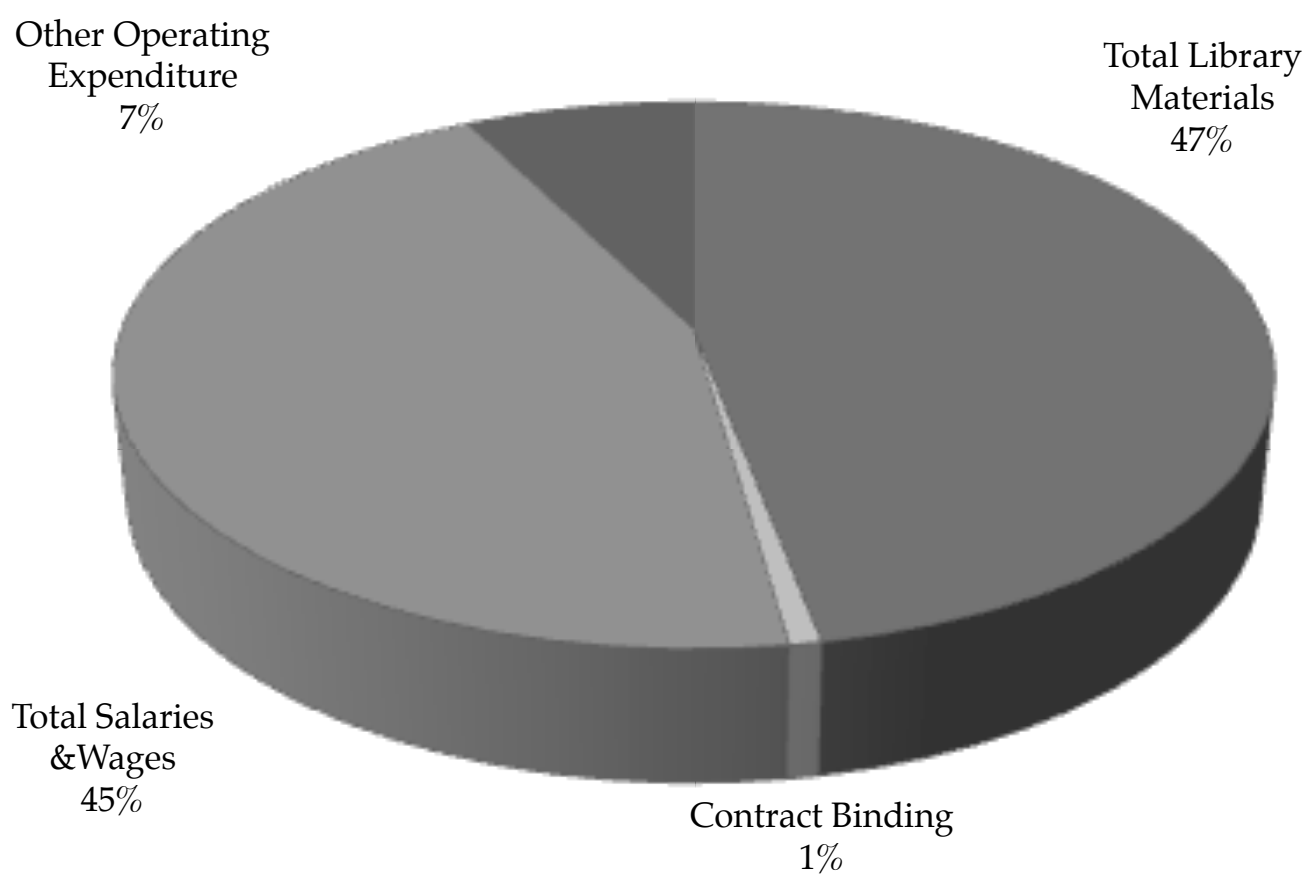

\footnotetext{
${ }^{1} 80$ ARL university libraries included data for a law library in the 2006-2007 ARL Statistics; among them, Alberta, British Columbia, Laval, McGill, Manitoba, and Wayne State did not complete this survey. Alberta and McGill completed this survey in the past, but chose not to complete this survey in 2006-2007. Colorado and Indiana completed this survey but did not include a law library in the ARL Statistics. Rutgers has two administratively independent law libraries, which respond separately to this survey; they are aggregated together in the ARL Statistics.

This figure includes Canadian universities, whose expenditures were converted to US dollars at the rate of 1.1323 Can\$ = 1 US\$, the average monthly noon exchange rate published in the Bank of Canada Review for the period July 2006 to June 2007.
} 

ARL Academic Law Library Data Tables 2006-2007 


\section{Collections Part 1}

Volumes Added (Gross)
Volumes Added (Net)
Monographs Purchased

(Volumes)
Current Serials Total

(Survey Question Number)

(1)

(1b.i)

(1b)

(2)

\begin{tabular}{|c|c|c|c|c|c|c|}
\hline INSTITUTION & Notes & & & & & \\
\hline ALABAMA & bG+ & 328,668 & 7,793 & 6,103 & 5,000 & 3,415 \\
\hline ARIZONA & G+ & 225,943 & 7,099 & $-35,408$ & 1,171 & 2,891 \\
\hline ARIZONA STATE & G & 272,173 & 4,853 & 2,968 & 804 & UA/NA \\
\hline BOSTON & + & 332,247 & 5,690 & 4,271 & 1,621 & 4,748 \\
\hline BOSTON COLLEGE & bG+ & 245,824 & 6,232 & 4,867 & 5,607 & 3,894 \\
\hline BRIGHAM YOUNG & bG+ & 337,197 & 7,258 & 2,462 & 1,582 & 4,264 \\
\hline CALIFORNIA, BERKELEY & G & 682,682 & 9,677 & 6,582 & UA/NA & 6,626 \\
\hline CALIFORNIA, DAVIS & G+ & 294,938 & 5,650 & 4,195 & 3,005 & 5,227 \\
\hline CALIFORNIA, LOS ANGELES & $\mathrm{G}+$ & 556,619 & 10,454 & 8,499 & 8,006 & 8,283 \\
\hline CASE WESTERN RESERVE & G+ & 306,226 & 5,785 & 2,064 & 953 & 5,957 \\
\hline CHICAGO & $\mathrm{G}+$ & 561,065 & 13,588 & $-25,020$ & 5,332 & 8,380 \\
\hline CINCINNATI & $\mathrm{G}^{+}$ & 284,250 & 4,744 & 71 & 1,736 & 6,627 \\
\hline COLORADO & G & 331,485 & 9,700 & 9,483 & 1,603 & 4,513 \\
\hline COLUMBIA & bG+ & 903,410 & 12,850 & 11,021 & 4,448 & 6,773 \\
\hline CONNECTICUT & G & 344,598 & 7,191 & 4,496 & 899 & 6,173 \\
\hline CORNELL & $\mathrm{bG}+$ & 564,386 & 8,670 & 8,377 & 906 & 25,000 \\
\hline DUKE & + & 442,021 & 10,152 & -28 & 2,744 & 3,411 \\
\hline EMORY & + & 217,631 & 11,111 & -700 & 1,073 & 9,841 \\
\hline FLORIDA & $\mathrm{G}^{+}$ & 322,990 & 4,976 & 3,035 & 1,511 & 8,067 \\
\hline FLORIDA STATE & $\mathrm{G}+$ & 307,797 & 3,680 & 2,843 & 781 & 4,800 \\
\hline GEORGE WASHINGTON & $\mathrm{bG}+$ & 356,932 & 8,857 & 6,659 & 6,659 & 21,102 \\
\hline GEORGETOWN & $\mathrm{bG}+$ & 562,986 & 21,927 & 19,373 & 4,737 & 8,672 \\
\hline GEORGIA & + & 623,325 & 10,678 & 9,204 & 119,434 & 32,818 \\
\hline HARVARD & + & $1,765,543$ & 25,709 & 21,466 & UA/NA & 15,725 \\
\hline HAWAII & G & 132,888 & 3,263 & 2,876 & 424 & 6,262 \\
\hline HOUSTON & bG+ & 523,350 & 13,722 & $-10,371$ & 12,205 & 2,221 \\
\hline HOWARD & $\mathrm{bG}+$ & 209,500 & 2,378 & 616 & 448 & 4,321 \\
\hline ILLINOIS, URBANA & $\mathrm{bG}+$ & 622,275 & 10,643 & 8,528 & 200 & 4,055 \\
\hline INDIANA & $\mathrm{G}^{+}$ & 449,617 & 13,433 & 10,232 & 7,502 & 11,927 \\
\hline IOWA & G+ & 798,317 & 22,389 & 18,816 & 16,200 & 10,101 \\
\hline KANSAS & + & 277,564 & 3,573 & $-36,683$ & 36,868 & 3,526 \\
\hline KENTUCKY & G & 261,953 & 3,862 & 2,352 & 2,879 & 5,650 \\
\hline LOUISIANA STATE & G+ & 452,841 & 11,772 & 9,637 & 1,123 & 6,835 \\
\hline LOUISVILLE & G & 238,703 & 2,015 & 2,015 & 2,015 & 5,420 \\
\hline MIAMI & G+ & 441,835 & 16,868 & 8,922 & 2,431 & 8,032 \\
\hline MICHIGAN & G+ & 700,417 & 14,817 & 10,335 & 3,442 & 6,263 \\
\hline MICHIGAN STATE & bG+ & 131,914 & 5,380 & 2,859 & 1,143 & 12,494 \\
\hline MINNESOTA & $\mathrm{bG}+$ & 730,399 & 17,140 & 16,207 & 2,038 & UA/NA \\
\hline MISSOURI & bG+ & 389,299 & 10,966 & 9,339 & 2,116 & 213 \\
\hline MONTREAL & G+ & 181,968 & 3,554 & 2,033 & 1,121 & 2,793 \\
\hline NEBRASKA & $\mathrm{G}+$ & 243,926 & 5,694 & 5,199 & UA/NA & 2,724 \\
\hline NEW MEXICO & G & 236,734 & 1,189 & 1,142 & 1,172 & 3,296 \\
\hline
\end{tabular}




\section{Collections Part 1}

Current Serials

Purchased

(4a)
Current Serials

Purchased

Electronic
Current Serials

Purchased Print
Current Serials

Not Purchased
Current Serials

Not Purchased Electronic
Current Serials

Not Purchased
(4b)
(4b.i)
(4b.ii) (Survey Question Number)

INSTITUTION

\begin{tabular}{|c|c|c|c|c|c|c|}
\hline 3,200 & 3,200 & 0 & 215 & 215 & 0 & ALABAMA \\
\hline 2,427 & 1,154 & 1,273 & 464 & 20 & 444 & ARIZONA \\
\hline 0 & 0 & 0 & 0 & 0 & 0 & ARIZONA STATE \\
\hline 4,671 & 2,144 & 2,527 & 77 & 7 & 70 & BOSTON \\
\hline 3,742 & 1,087 & 2,655 & 152 & 6 & 146 & BOSTON COLLEGE \\
\hline 3,999 & 1 & 3,998 & 265 & 0 & 265 & BRIGHAM YOUNG \\
\hline 5,769 & 1,617 & 4,152 & 857 & 40 & 817 & CALIFORNIA, BERKELEY \\
\hline 4,533 & 1,040 & 3,493 & 694 & 49 & 645 & CALIFORNIA, DAVIS \\
\hline UA/NA & UA/NA & $\mathrm{UA} / \mathrm{NA}$ & UA/NA & $\mathrm{UA} / \mathrm{NA}$ & $\mathrm{UA} / \mathrm{NA}$ & CALIFORNIA, LOS ANGELES \\
\hline 5,353 & 4,560 & 793 & 604 & 0 & 604 & CASE WESTERN RESERVE \\
\hline $\mathrm{UA} / \mathrm{NA}$ & UA/NA & UA/NA & $\mathrm{UA} / \mathrm{NA}$ & $\mathrm{UA} / \mathrm{NA}$ & $\mathrm{UA} / \mathrm{NA}$ & CHICAGO \\
\hline 6,587 & 0 & 6,587 & 40 & 0 & 40 & CINCINNATI \\
\hline 4,513 & 920 & 3,593 & 0 & 0 & 0 & COLORADO \\
\hline 5,854 & 1,592 & 4,262 & 919 & 0 & 919 & COLUMBIA \\
\hline 5,265 & 1,425 & 3,840 & 908 & 338 & 570 & CONNECTICUT \\
\hline UA/NA & UA/NA & UA/NA & UA/NA & UA/NA & UA/NA & CORNELL \\
\hline 3,257 & 36 & 3,221 & 154 & 8 & 146 & DUKE \\
\hline 9,770 & 5,974 & 3,796 & 71 & 0 & 71 & EMORY \\
\hline 8,067 & 0 & 8,067 & 0 & 0 & 0 & FLORIDA \\
\hline 3,866 & 2,258 & 1,608 & 934 & 0 & 934 & FLORIDA STATE \\
\hline 21,102 & 12,725 & 8,377 & 0 & 0 & 0 & GEORGE WASHINGTON \\
\hline 8,593 & 747 & 7,846 & 79 & 79 & 0 & GEORGETOWN \\
\hline 31,879 & 29,363 & 2,516 & 939 & 920 & 19 & GEORGIA \\
\hline UA/NA & UA/NA & UA/NA & UA/NA & UA/NA & UA/NA & HARVARD \\
\hline 5,783 & 3,378 & 2,405 & 479 & 242 & 237 & HAWAII \\
\hline 2,030 & 44 & 1,986 & 191 & 8 & 183 & HOUSTON \\
\hline 4,306 & 3,154 & 1,152 & 15 & 0 & 15 & HOWARD \\
\hline 3,921 & 11 & 3,910 & 134 & 0 & 134 & ILLINOIS, URBANA \\
\hline 11,319 & 1,414 & 9,905 & 608 & 533 & 75 & INDIANA \\
\hline 8,081 & 1,616 & 6,465 & 2,020 & 606 & 1,414 & IOWA \\
\hline 3,526 & 1,587 & 1,939 & 0 & 0 & 0 & KANSAS \\
\hline 5,502 & 4,250 & 1,252 & 148 & 0 & 148 & KENTUCKY \\
\hline 6,817 & 4,988 & 1,829 & 18 & 0 & 18 & LOUISIANA STATE \\
\hline 5,420 & 0 & 5,420 & 0 & UA/NA & UA/NA & LOUISVILLE \\
\hline 8,011 & 7,703 & 308 & 21 & 21 & 0 & MIAMI \\
\hline 6,129 & $\mathrm{UA} / \mathrm{NA}$ & UA/NA & 134 & UA/NA & $\mathrm{UA} / \mathrm{NA}$ & MICHIGAN \\
\hline 12,494 & 10,211 & 2,283 & 0 & 0 & 0 & MICHIGAN STATE \\
\hline $\mathrm{UA} / \mathrm{NA}$ & $\mathrm{UA} / \mathrm{NA}$ & UA/NA & UA/NA & UA/NA & $\mathrm{UA} / \mathrm{NA}$ & MINNESOTA \\
\hline 0 & 0 & 0 & 213 & 213 & 0 & MISSOURI \\
\hline 2,705 & 1,684 & 1,021 & 88 & 0 & 88 & MONTREAL \\
\hline 2,522 & 2,522 & 0 & 202 & 0 & 202 & NEBRASKA \\
\hline 3,295 & 860 & 2,435 & 1 & 0 & 1 & NEW MEXICO \\
\hline
\end{tabular}




\section{Collections Part 1}

Current Serials

Purchased

(4a)
Current Serials

Purchased

Electronic
Current Serials

Purchased Print
Current Serials

Not Purchased
Current Serials

Not Purchased Electronic
Current Serials

Not Purchased
(4b)
(4b.i)

\begin{tabular}{|c|c|c|c|c|c|c|}
\hline 4,226 & 0 & 4,226 & 820 & 0 & 820 & NEW YORK \\
\hline 5,881 & 1,551 & 4,330 & 1,051 & 69 & 982 & NORTH CAROLINA \\
\hline $\mathrm{UA} / \mathrm{NA}$ & UA/NA & UA/NA & UA/NA & $\mathrm{UA} / \mathrm{NA}$ & $\mathrm{UA} / \mathrm{NA}$ & NORTHWESTERN \\
\hline 35,074 & 32,785 & 2,289 & 1,993 & UA/NA & UA/NA & NOTRE DAME \\
\hline 7,283 & 4,949 & 2,334 & 1,170 & 0 & 1,170 & OHIO STATE \\
\hline $\mathrm{UA} / \mathrm{NA}$ & $\mathrm{UA} / \mathrm{NA}$ & $\mathrm{UA} / \mathrm{NA}$ & $\mathrm{UA} / \mathrm{NA}$ & $\mathrm{UA} / \mathrm{NA}$ & UA/NA & OKLAHOMA \\
\hline 1,670 & 0 & 1,670 & 62 & 0 & 62 & OREGON \\
\hline 40,581 & 33,091 & 7,490 & 830 & 79 & 751 & PENNSYLVANIA \\
\hline 7,216 & 1,908 & 5,308 & 0 & 0 & 0 & PENNSYLVANIA STATE \\
\hline 3,818 & 1,474 & 2,344 & 285 & 127 & 158 & PITTSBURGH \\
\hline $\mathrm{UA} / \mathrm{NA}$ & UA/NA & $\mathrm{UA} / \mathrm{NA}$ & $\mathrm{UA} / \mathrm{NA}$ & $\mathrm{UA} / \mathrm{NA}$ & UA/NA & QUEENS \\
\hline 5,116 & 4,466 & 650 & 0 & 0 & 0 & RUTGERS-Camden \\
\hline 4,911 & 4,466 & 445 & 0 & 0 & 0 & RUTGERS \\
\hline 1,890 & 1,364 & 526 & 166 & 143 & 23 & SASKATCHEWAN \\
\hline 3,126 & 0 & 3,126 & 0 & 0 & 0 & SOUTH CAROLINA \\
\hline 6,396 & 1,739 & 4,657 & 1,675 & 186 & 1,489 & SOUTHERN CALIFORNIA \\
\hline 1,533 & 120 & 1,413 & 0 & 0 & 0 & SOUTHERN ILLINOIS \\
\hline 2,556 & 0 & 2,556 & 3,103 & 0 & 3,103 & SUNY-BUFFALO \\
\hline 2,190 & 1,241 & 949 & 0 & 0 & 0 & SYRACUSE \\
\hline 3,014 & 1,356 & 1,658 & 437 & 284 & 153 & TEMPLE \\
\hline 2,114 & 669 & 1,445 & 0 & UA/NA & $\mathrm{UA} / \mathrm{NA}$ & TENNESSEE \\
\hline 4,598 & 0 & 4,598 & 2,593 & 1,685 & 908 & TEXAS \\
\hline 3,909 & 3,471 & 438 & 804 & 730 & 74 & TEXAS TECH \\
\hline 1,873 & 0 & 1,873 & 29 & 29 & 0 & TORONTO \\
\hline 5,277 & 0 & 5,277 & 0 & 0 & 0 & TULANE \\
\hline $\mathrm{UA} / \mathrm{NA}$ & UA/NA & UA/NA & UA/NA & UA/NA & UA/NA & UTAH \\
\hline 6,867 & 5,487 & 1,380 & 0 & 0 & 0 & VANDERBILT \\
\hline UA/NA & UA/NA & UA/NA & UA/NA & UA/NA & UA/NA & VIRGINIA \\
\hline UA/NA & UA/NA & UA/NA & UA/NA & UA/NA & UA/NA & WASHINGTON \\
\hline 1,006 & 150 & 856 & 3,223 & 577 & 2,646 & WASHINGTON U.-ST. LOUIS \\
\hline 1,075 & 0 & 1,075 & 325 & 0 & 325 & WESTERN ONTARIO \\
\hline 3,117 & 0 & 3,117 & 2,957 & 0 & 2,957 & WISCONSIN \\
\hline 9,556 & 123 & 9,433 & 302 & 0 & 302 & YALE \\
\hline \multicolumn{7}{|c|}{ SumMary Data } \\
\hline 40,581 & 33,091 & 9,905 & 3,223 & 1,685 & 3,103 & High \\
\hline 6,616 & 4,361 & 3,227 & 697 & 277 & 588 & Mean \\
\hline 4,635 & 1,617 & 2,516 & 314 & 135 & 237 & Median \\
\hline 1,006 & 1 & 308 & 1 & 6 & 1 & Low \\
\hline 410,181 & 213,675 & 190,377 & 33,469 & 7,214 & 24,128 & Sum \\
\hline 62 & 49 & 59 & 48 & 26 & 41 & Number of Libraries \\
\hline
\end{tabular}




\section{Collections Part 2}

Microform Government Computer Manuscripts Cartographic Units Documents
Files and Archives Materials
Graphic

Materials
Audio
Materials

(11)

(12)
Film and

(6)

(7)

(8)

(9)

(10)

(13)

\begin{tabular}{|c|c|c|c|c|c|c|c|c|c|}
\hline INSTITUTION & Notes & & & & & & & & \\
\hline ALABAMA & bG+ & 784,971 & 421,716 & 600 & 1,300 & 0 & 0 & 644 & 1,353 \\
\hline ARIZONA & G+ & 688,532 & 0 & 482 & 0 & 9 & 0 & 306 & 222 \\
\hline ARIZONA STATE & G & 724,843 & 147,714 & 1,461 & 0 & 0 & 0 & 126 & 460 \\
\hline BOSTON & + & $1,672,358$ & 0 & 55 & 93 & 0 & 0 & 63 & 68 \\
\hline BOSTON COLLEGE & bG+ & $1,505,297$ & 4,285 & 398 & 649 & 0 & 0 & 50 & 425 \\
\hline BRIGHAM YOUNG & bG+ & 826,143 & UA/NA & UA/NA & 0 & UA/NA & $\mathrm{UA} / \mathrm{NA}$ & 1,395 & $\mathrm{UA} / \mathrm{NA}$ \\
\hline CALIFORNIA, BERKELEY & G & 932,346 & 0 & 252 & 297 & 1 & 2,500 & 2 & 189 \\
\hline CALIFORNIA, DAVIS & G+ & 738,363 & 39,196 & 411 & 51 & 20 & 0 & 798 & 147 \\
\hline CALIFORNIA, LOS ANGELES & G+ & 420,125 & 0 & 854 & 6 & 2 & 0 & 799 & 1,564 \\
\hline CASE WESTERN RESERVE & G+ & 541,803 & 2,097 & 300 & 107 & 0 & 0 & 163 & 2,383 \\
\hline CHICAGO & $\mathrm{G}^{+}$ & 332,521 & $\mathrm{UA} / \mathrm{NA}$ & 426 & UA/NA & 0 & 0 & 462 & 409 \\
\hline CINCINNATI & G+ & 745,327 & 0 & 245 & 434 & 0 & 0 & 933 & 356 \\
\hline COLORADO & G & $1,596,771$ & 0 & 692 & 88 & 2 & 0 & 431 & 1,057 \\
\hline COLUMBIA & bG+ & 900,085 & 0 & 1,291 & 2,892 & 32 & 1 & 145 & 295 \\
\hline CONNECTICUT & G & $1,113,037$ & 0 & 507 & 122 & 0 & 0 & 779 & 351 \\
\hline CORNELL & bG+ & 975,051 & $\mathrm{UA} / \mathrm{NA}$ & 39 & 0 & 0 & 0 & 360 & 225 \\
\hline DUKE & + & $1,172,484$ & 0 & 194 & 86 & 0 & 0 & 273 & 299 \\
\hline EMORY & + & 607,189 & 47,278 & 169 & 62 & 5 & 0 & 75 & 405 \\
\hline FLORIDA & $\mathrm{G}^{+}$ & $1,642,972$ & $\mathrm{UA} / \mathrm{NA}$ & 41 & UA/NA & 9 & 0 & 17 & 1,177 \\
\hline FLORIDA STATE & $\mathrm{G}^{+}$ & $1,050,392$ & 0 & 449 & 0 & 0 & 242 & 1,393 & 3,338 \\
\hline GEORGE WASHINGTON & bG+ & $1,380,733$ & 0 & 110 & 115 & 0 & 0 & 235 & 250 \\
\hline GEORGETOWN & bG+ & $2,619,073$ & 9,385 & 1,204 & 3,869 & 85 & 501 & 5,701 & 1,020 \\
\hline GEORGIA & + & 616,237 & 937,229 & 1,471 & 147 & 1,499 & 0 & 384 & 341 \\
\hline HARVARD & + & $2,100,091$ & $\mathrm{UA} / \mathrm{NA}$ & UA/NA & UA/NA & UA/NA & UA/NA & UA/NA & $\mathrm{UA} / \mathrm{NA}$ \\
\hline HAWAII & G & $1,193,169$ & 0 & 389 & 0 & 0 & $\mathrm{UA} / \mathrm{NA}$ & 161 & 354 \\
\hline HOUSTON & bG+ & $1,591,069$ & 0 & 0 & 0 & 0 & 0 & 2 & 30 \\
\hline HOWARD & bG+ & 418,117 & 2,205 & 27 & 800 & 0 & 0 & 198 & 575 \\
\hline ILLINOIS, URBANA & bG+ & 928,746 & 0 & 45 & 0 & 0 & 0 & 0 & 42 \\
\hline INDIANA & G+ & $1,642,727$ & 0 & 670 & 647 & 0 & 1,054 & 920 & 1,305 \\
\hline IOWA & $\mathrm{G}^{+}$ & $1,736,819$ & $\mathrm{UA} / \mathrm{NA}$ & 2,971 & UA/NA & 3,505 & 11 & 1,001 & 1,177 \\
\hline KANSAS & + & 532,536 & 9,021 & 192 & 10 & 3 & 0 & 63 & 168 \\
\hline KENTUCKY & G & $1,218,726$ & 0 & 190 & 0 & 0 & 0 & 0 & 490 \\
\hline LOUISIANA STATE & G+ & $2,230,841$ & $\mathrm{UA} / \mathrm{NA}$ & 1,520 & 20 & 752 & 12 & 2,933 & 1,370 \\
\hline LOUISVILLE & G & UA/NA & UA/NA & UA/NA & UA/NA & UA/NA & $\mathrm{UA} / \mathrm{NA}$ & UA/NA & $\mathrm{UA} / \mathrm{NA}$ \\
\hline MIAMI & $\mathrm{G}^{+}$ & 917,140 & 0 & 1,278 & 0 & 0 & 0 & 1,163 & 860 \\
\hline MICHIGAN & G+ & $1,626,832$ & 0 & 57 & 0 & 0 & 0 & 101 & 128 \\
\hline MICHIGAN STATE & $\mathrm{bG}^{+}$ & 920,706 & $\mathrm{UA} / \mathrm{NA}$ & UA/NA & UA/NA & UA/NA & $\mathrm{UA} / \mathrm{NA}$ & 341 & 676 \\
\hline MINNESOTA & bG+ & $2,019,444$ & 0 & 263 & 172 & 3 & UA/NA & $\mathrm{UA} / \mathrm{NA}$ & UA/NA \\
\hline MISSOURI & bG+ & 591,120 & $\mathrm{UA} / \mathrm{NA}$ & 0 & 0 & 0 & 0 & 0 & 947 \\
\hline MONTREAL & G+ & 66,998 & 0 & 214 & 0 & 0 & 5 & 249 & 110 \\
\hline NEBRASKA & $\mathrm{G}^{+}$ & $1,034,514$ & $\mathrm{UA} / \mathrm{NA}$ & UA/NA & UA/NA & 0 & 0 & 508 & 412 \\
\hline NEW MEXICO & G & 915,704 & UA/NA & 198 & UA/NA & UA/NA & UA/NA & UA/NA & UA/NA \\
\hline
\end{tabular}




\section{Collections Part 2}

Microform Government Computer Manuscripts Cartographic Units Documents
Files and Archives Materials
Graphic

Materials
Audio

Materials
Film and

(6)

(7)

(8)

(9)

(10)

(11)

(12)

(13)

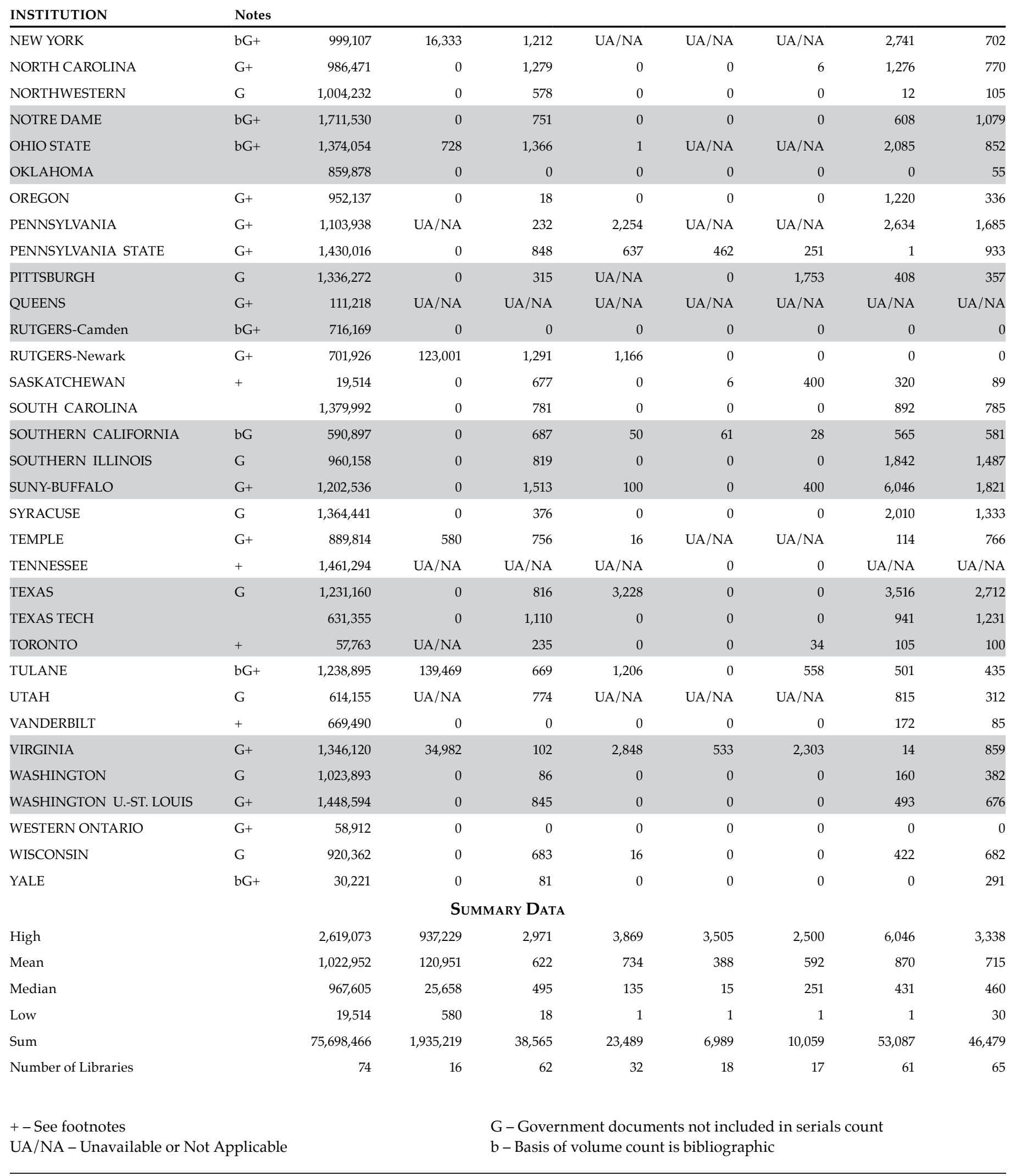




\section{EXPENDITURES}

Monographs Current Serials Other Library Misc. Materials Total Library Contract Binding Materials Materials

(Survey Question Number)

(15a)

(15b)

(15c)

(15d)

(15)

(16)

\begin{tabular}{|c|c|c|c|c|c|c|c|}
\hline INSTITUTION & Notes & & & & & & \\
\hline ALABAMA & bG+ & 162,432 & $1,000,413$ & 206,181 & 0 & $1,369,026$ & 20,000 \\
\hline ARIZONA & G+ & 61,742 & 717,641 & 309,214 & 12,979 & $1,101,576$ & 6,190 \\
\hline ARIZONA STATE & G & 36,617 & 907,588 & 5,860 & 4,000 & 954,065 & 11,346 \\
\hline BOSTON & + & 99,415 & $1,135,901$ & 0 & 0 & $1,235,316$ & 22,300 \\
\hline BOSTON COLLEGE & bG+ & 133,985 & 932,923 & 207,981 & UA/NA & $1,274,889$ & 17,494 \\
\hline BRIGHAM YOUNG & bG+ + & 59,444 & 753,143 & 10,249 & 0 & 822,836 & 10,466 \\
\hline CALIFORNIA, BERKELEY & G & 598,949 & $1,622,153$ & 278,518 & 0 & $2,499,620$ & 38,905 \\
\hline CALIFORNIA, DAVIS & G+ & 193,544 & 774,595 & UA/NA & 0 & 968,139 & 25,294 \\
\hline CALIFORNIA, LOS ANGELES & G+ & 217,990 & $1,609,061$ & UA/NA & UA/NA & $1,827,051$ & 44,770 \\
\hline CASE WESTERN RESERVE & G+ & 60,962 & $1,054,574$ & 206,705 & 6,429 & $1,328,670$ & 11,703 \\
\hline CHICAGO & G+ & 549,606 & $1,421,949$ & 14,579 & UA/NA & $1,986,134$ & 53,357 \\
\hline CINCINNATI & G+ & 55,178 & 802,670 & 7,532 & 7,741 & 873,121 & 6,608 \\
\hline COLORADO & G & 97,816 & 893,148 & 327,442 & 0 & $1,318,406$ & 13,568 \\
\hline COLUMBIA & bG+ & 380,774 & $1,811,826$ & 0 & 0 & $2,192,600$ & 61,543 \\
\hline CONNECTICUT & G & 105,664 & $1,230,044$ & 19,021 & 100,453 & $1,455,182$ & 29,489 \\
\hline CORNELL & bG+ + & 934,261 & 479,048 & 143 & 34,108 & $1,447,560$ & 22,754 \\
\hline DUKE & + & 234,915 & $1,331,183$ & 53,376 & 88,946 & $1,708,420$ & 17,264 \\
\hline EMORY & + & 67,287 & 825,656 & 8,000 & 249,161 & $1,150,104$ & 5,799 \\
\hline FLORIDA & G+ & 193,890 & 688,138 & 347,964 & 23,355 & $1,253,347$ & 17,102 \\
\hline FLORIDA STATE & G+ & 84,969 & 698,522 & 3,138 & 91,147 & 877,776 & 5,446 \\
\hline GEORGE WASHINGTON & bG+ & $1,585,954$ & $1,205,028$ & 0 & 0 & $2,790,982$ & 66,517 \\
\hline GEORGETOWN & bG+ & 323,613 & $2,179,630$ & 196,691 & 73,869 & $2,773,803$ & 59,256 \\
\hline GEORGIA & + & 99,570 & 919,420 & 407,815 & 21,026 & $1,447,831$ & 13,361 \\
\hline HARVARD & + & 696,630 & $2,500,173$ & 535,850 & 1,021 & $3,733,674$ & 177,361 \\
\hline HAWAII & G & 16,974 & 467,335 & 86,649 & 86,001 & 656,959 & 3,013 \\
\hline HOUSTON & bG+ & 94,176 & 863,619 & 23,914 & 8 & 981,717 & 8,854 \\
\hline HOWARD & bG+ & 51,445 & 525,955 & 0 & 0 & 577,400 & 3,000 \\
\hline ILLINOIS, URBANA & bG+ & 14,380 & 992,381 & 0 & 0 & $1,006,761$ & $\mathrm{UA} / \mathrm{NA}$ \\
\hline INDIANA & G+ & 72,750 & $1,115,412$ & 333,931 & 9,759 & $1,531,852$ & 27,293 \\
\hline IOWA & G+ & 629,698 & $1,858,455$ & 164,546 & 0 & $2,652,699$ & 30,008 \\
\hline KANSAS & + & 62,716 & 493,466 & 0 & 1,721 & 557,903 & 6,500 \\
\hline KENTUCKY & G & 7,311 & $1,049,766$ & 0 & 21,362 & $1,078,439$ & 5,531 \\
\hline LOUISIANA STATE & G+ & 131,173 & $1,027,807$ & 40,836 & 0 & $1,199,816$ & 14,728 \\
\hline LOUISVILLE & G & 255,588 & $1,033,379$ & 45,426 & UA/NA & $1,334,393$ & 17,246 \\
\hline MIAMI & G+ & 160,677 & $1,770,475$ & 0 & 357,162 & $2,288,314$ & 20,505 \\
\hline MICHIGAN & $\mathrm{G}^{+}$ & 183,899 & $2,129,989$ & 84,392 & 51,095 & $2,449,375$ & 64,079 \\
\hline MICHIGAN STATE & bG+ & 110,486 & 501,057 & 377,197 & 2,728 & 991,468 & 7,349 \\
\hline MINNESOTA & $\mathrm{bG}+$ & 286,833 & $1,263,621$ & 132,668 & 35,873 & $1,718,995$ & 48,144 \\
\hline MISSOURI & bG+ & 124,831 & 400,473 & 8,664 & 0 & 533,968 & 4,663 \\
\hline MONTREAL & G+ & 107,630 & 592,849 & 0 & 0 & 700,479 & 7,535 \\
\hline NEBRASKA & G+ & 26,412 & 519,187 & 19,475 & 6,910 & 571,984 & 9,115 \\
\hline NEW MEXICO & G & 44,177 & 364,597 & 70 & UA/NA & 408,844 & 3,211 \\
\hline
\end{tabular}




\section{EXPENDITURES}

Salaries \& Wages Professional Staff
Salaries \& Wages Salaries \& Wages Support Staff Student Assistants
Salaries \& Wages

Total
Other Operating

Expenditures
Total Library

Expenditures (17a)

(17b)
$(17 \mathrm{c})$
(17)
(19)

(20) (Survey Question Number)

INSTITUTION

\begin{tabular}{|c|c|c|c|c|c|c|}
\hline 646,240 & 200,110 & 62,638 & 908,988 & 117,416 & $2,415,430$ & ALABAMA \\
\hline 654,904 & 304,978 & 17,613 & 977,495 & 44,665 & $2,129,926$ & ARIZONA \\
\hline 481,562 & 386,529 & 22,838 & 890,929 & 62,968 & $1,919,308$ & ARIZONA STATE \\
\hline 890,969 & 267,338 & 73,792 & $1,232,099$ & 405,442 & $2,895,157$ & BOSTON \\
\hline $1,026,530$ & 363,319 & 52,531 & $1,442,380$ & 188,973 & $2,923,736$ & BOSTON COLLEGE \\
\hline 654,261 & 95,191 & 227,835 & 977,287 & 111,922 & $1,922,511$ & BRIGHAM YOUNG \\
\hline $1,336,066$ & $1,255,543$ & 313,778 & $2,905,387$ & 712,169 & $6,156,081$ & CALIFORNIA, BERKELEY \\
\hline 608,312 & 377,662 & 13,137 & 999,111 & 102,864 & $2,095,408$ & CALIFORNIA, DAVIS \\
\hline $1,136,016$ & 470,317 & 95,383 & $1,701,716$ & 375,456 & $3,948,993$ & CALIFORNIA, LOS ANGELES \\
\hline 851,755 & 337,202 & 109,551 & $1,298,508$ & 176,695 & $2,815,576$ & CASE WESTERN RESERVE \\
\hline 833,199 & 491,741 & 60,998 & $1,385,938$ & 79,542 & $3,504,971$ & CHICAGO \\
\hline 480,840 & 247,401 & 47,900 & 776,141 & 130,033 & $1,785,903$ & CINCINNATI \\
\hline 609,176 & 344,570 & 62,312 & $1,016,058$ & 118,406 & $2,466,438$ & COLORADO \\
\hline $1,375,015$ & 831,219 & 94,778 & $2,301,012$ & 407,564 & $4,962,719$ & COLUMBIA \\
\hline 798,177 & 452,127 & 63,815 & $1,314,119$ & 232,412 & $3,031,202$ & CONNECTICUT \\
\hline 686,479 & 445,871 & 39,852 & $1,172,202$ & 76,954 & $2,719,470$ & CORNELL \\
\hline 869,480 & 395,904 & 40,081 & $1,305,465$ & 86,116 & $3,117,265$ & DUKE \\
\hline 666,235 & 277,632 & 78,193 & $1,022,060$ & 352,880 & $2,530,843$ & EMORY \\
\hline 821,574 & 594,811 & 90,170 & $1,506,555$ & 6,466 & $2,783,470$ & FLORIDA \\
\hline 587,401 & 184,821 & 74,114 & 846,336 & 68,673 & $1,798,231$ & FLORIDA STATE \\
\hline $1,596,477$ & 686,500 & 165,786 & $2,448,763$ & 161,456 & $5,467,718$ & GEORGE WASHINGTON \\
\hline $1,894,627$ & $1,583,807$ & 280,468 & $3,758,902$ & 702,309 & $7,294,270$ & GEORGETOWN \\
\hline 503,310 & 258,499 & 46,881 & 808,690 & 301,891 & $2,571,773$ & GEORGIA \\
\hline $3,547,004$ & $2,152,394$ & 83,553 & $5,782,951$ & 957,653 & $10,651,639$ & HARVARD \\
\hline 436,583 & 88,527 & 211,558 & 736,668 & 221,139 & $1,617,779$ & HAWAII \\
\hline 638,947 & 232,096 & 101,948 & 972,991 & 164,568 & $2,128,130$ & HOUSTON \\
\hline 531,026 & 251,435 & 58,278 & 840,739 & 11,708 & $1,432,847$ & HOWARD \\
\hline 629,068 & 269,260 & 29,411 & 927,739 & $\mathrm{UA} / \mathrm{NA}$ & $1,934,500$ & ILLINOIS, URBANA \\
\hline 757,714 & 238,578 & 60,305 & $1,056,597$ & 15,683 & $2,631,425$ & INDIANA \\
\hline 977,419 & 656,309 & 101,773 & $1,735,501$ & 204,917 & $4,623,125$ & IOWA \\
\hline 376,677 & 213,036 & 37,113 & 626,826 & 70,870 & $1,262,099$ & KANSAS \\
\hline 288,906 & 156,733 & 39,843 & 485,482 & 78,776 & $1,648,228$ & KENTUCKY \\
\hline 713,603 & 281,165 & 97,311 & $1,092,079$ & 157,354 & $2,463,977$ & LOUISIANA STATE \\
\hline 434,741 & 238,182 & 28,805 & 701,728 & 237,834 & $2,291,201$ & LOUISVILLE \\
\hline 655,582 & 395,947 & 65,876 & $1,117,405$ & 284,740 & $3,710,964$ & MIAMI \\
\hline UA/NA & UA/NA & 289,706 & $2,314,690$ & 504,644 & $5,332,788$ & MICHIGAN \\
\hline 593,998 & 131,960 & 137,527 & 863,485 & 20,207 & $1,882,509$ & MICHIGAN STATE \\
\hline $1,076,548$ & 580,192 & 177,310 & $1,834,050$ & 369,208 & $3,970,397$ & MINNESOTA \\
\hline 394,708 & 248,765 & 60,263 & 703,736 & 28,501 & $1,270,868$ & MISSOURI \\
\hline 295,710 & 380,119 & 0 & 675,829 & 11,823 & $1,395,665$ & MONTREAL \\
\hline 356,946 & 150,479 & 48,065 & 555,490 & 34,907 & $1,171,496$ & NEBRASKA \\
\hline 497,362 & 321,137 & 41,979 & 860,478 & 658,581 & $1,931,114$ & NEW MEXICO \\
\hline
\end{tabular}




\section{EXPENDITURES}

Monographs

(Survey Question Number) (15a)
Current Serials

(15b)
Other Library
Materials

(15c)
Misc. Materials

(15d)
Total Library Contract Binding Materials

(15)
(16)

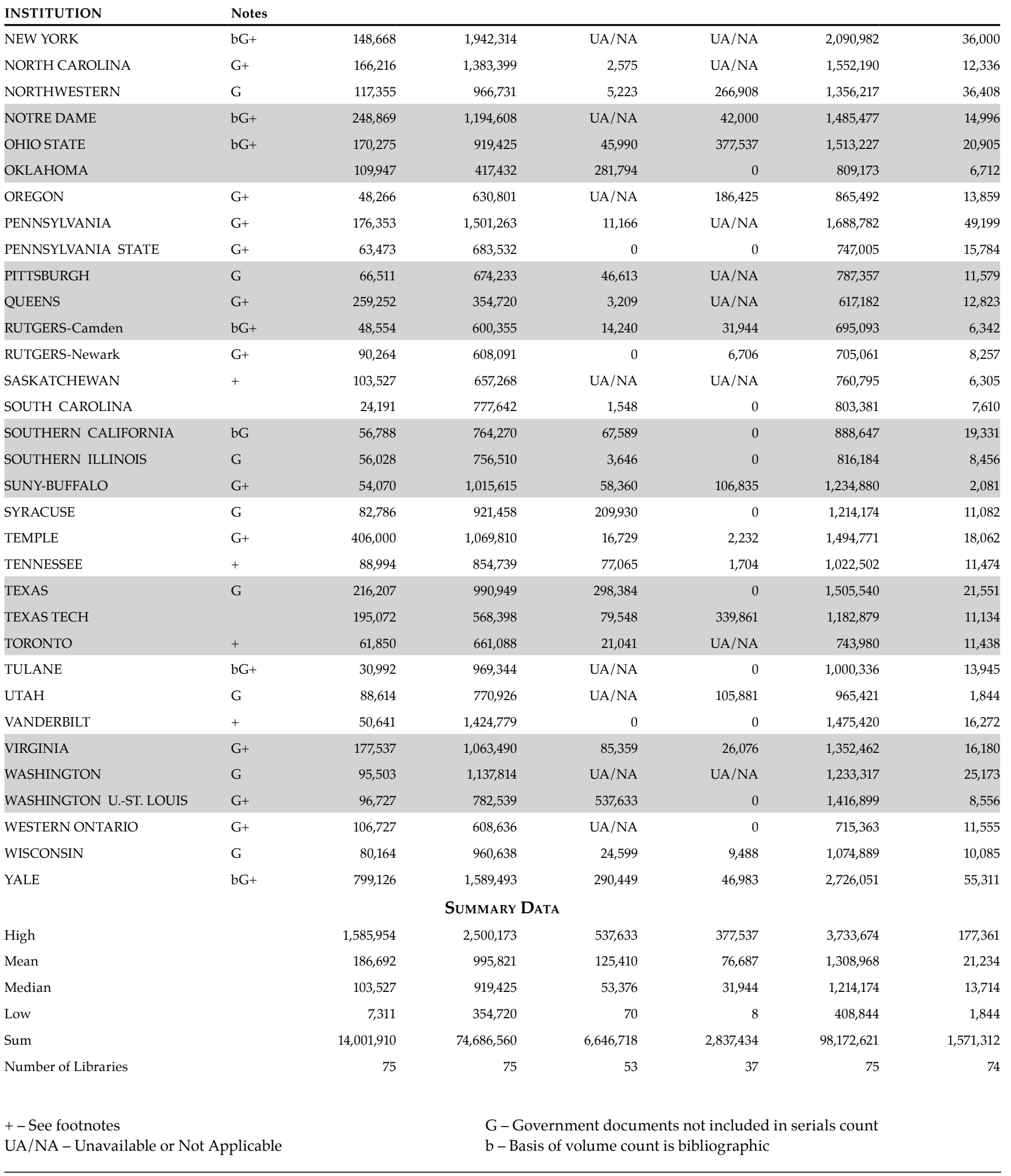

16 · ARL Academic Law Library Statistics 2006-2007 


\section{EXPENDITURES}

Salaries \& Wages Salaries \& Wages Salaries \& Wages Professional Staff Support Staff Student Assistants (17a) (17b) (17c)
Total Salaries \&Wages

(17)
Other Operating

Expenditures

(19)
Total Library

Expenditures

(20) (Survey Question Number)

\begin{tabular}{|c|c|c|c|c|c|c|}
\hline & & & & & & INSTITUTION \\
\hline $1,454,557$ & 991,809 & 73,015 & $2,519,381$ & 368,000 & $5,014,363$ & NEW YORK \\
\hline 743,931 & 297,834 & 74,799 & $1,116,564$ & 123,968 & $2,805,058$ & NORTH CAROLINA \\
\hline 687,053 & 621,771 & 31,270 & $1,340,094$ & 236,998 & $2,969,717$ & NORTHWESTERN \\
\hline 710,600 & 407,462 & 81,725 & $1,199,787$ & 537,089 & $3,237,349$ & NOTRE DAME \\
\hline 592,542 & 349,137 & 79,874 & $1,021,553$ & 82,644 & $2,638,329$ & OHIO STATE \\
\hline 437,768 & 164,984 & 47,977 & 650,729 & 87,320 & $1,553,934$ & OKLAHOMA \\
\hline 402,602 & 172,495 & 85,474 & 660,571 & 82,587 & $1,622,509$ & OREGON \\
\hline $1,094,107$ & 647,161 & 28,568 & $1,769,836$ & 164,575 & $3,672,392$ & PENNSYLVANIA \\
\hline 627,779 & 332,580 & 47,510 & $1,007,869$ & 79,445 & $1,850,103$ & PENNSYLVANIA STATE \\
\hline 470,110 & 215,586 & 23,490 & 709,186 & 357,022 & $1,865,144$ & PITTSBURGH \\
\hline 127,905 & 252,667 & 36,469 & 417,041 & 21,995 & $1,069,042$ & QUEENS \\
\hline 591,875 & 482,322 & 9,025 & $1,083,222$ & 27,802 & $1,812,459$ & RUTGERS \\
\hline 684,678 & 416,319 & 45,983 & $1,146,980$ & 126,027 & $1,986,325$ & RUTGERS \\
\hline 185,455 & 162,197 & 19,806 & 367,458 & UA/NA & $1,134,558$ & SASKATCHEWAN \\
\hline 508,678 & 248,925 & 18,669 & 776,272 & 360,625 & $1,947,888$ & SOUTH CAROLINA \\
\hline 862,809 & 485,458 & 83,530 & $1,431,797$ & 199,391 & $2,539,166$ & SOUTHERN CALIFORNIA \\
\hline 378,845 & 245,309 & 48,380 & 672,534 & 44,089 & $1,541,263$ & SOUTHERN ILLINOIS \\
\hline 737,352 & 217,699 & 127,446 & $1,082,497$ & 262,365 & $2,581,823$ & SUNY-BUFFALO \\
\hline 619,134 & 291,690 & 59,946 & 970,770 & 95,075 & $2,291,101$ & SYRACUSE \\
\hline 701,231 & 234,738 & 133,912 & $1,069,881$ & 257,123 & $2,839,837$ & TEMPLE \\
\hline 658,773 & 453,046 & 87,480 & $1,199,299$ & 186,345 & $2,419,620$ & TENNESSEE \\
\hline 890,145 & 652,541 & 46,556 & $1,589,242$ & 385,181 & $3,501,514$ & TEXAS \\
\hline 440,092 & 597,577 & 75,609 & $1,113,278$ & 377,023 & $2,684,314$ & TEXAS TECH \\
\hline 629,008 & 353,390 & 59,799 & $1,042,197$ & 105,419 & $1,903,034$ & TORONTO \\
\hline 254,807 & 200,508 & 57,018 & 512,333 & 184,658 & $1,711,272$ & TULANE \\
\hline 522,899 & 265,714 & 42,116 & 830,729 & 57,177 & $1,855,171$ & UTAH \\
\hline 385,510 & 310,764 & 43,270 & 739,544 & 193,680 & $2,424,916$ & VANDERBILT \\
\hline 917,000 & 577,848 & 47,047 & $1,541,895$ & 168,179 & $3,078,716$ & VIRGINIA \\
\hline $1,045,557$ & 524,937 & 81,658 & $1,652,152$ & 214,636 & $3,125,278$ & WASHINGTON \\
\hline 570,200 & 299,659 & 65,159 & 935,018 & 465,110 & $2,825,583$ & WASHINGTON U.-ST. LOUIS \\
\hline 188,193 & 110,435 & 9,428 & 308,055 & 9,564 & $1,044,537$ & WESTERN ONTARIO \\
\hline 873,913 & 308,995 & 135,984 & $1,318,892$ & 146,683 & $2,550,549$ & WISCONSIN \\
\hline $1,501,282$ & $1,130,203$ & 63,401 & $2,694,886$ & 640,000 & $6,116,248$ & YALE \\
\hline \multicolumn{7}{|c|}{ SUMMARY DATA } \\
\hline $3,547,004$ & $2,152,394$ & 313,778 & $5,782,951$ & 957,653 & $10,651,639$ & High \\
\hline 739,291 & 417,070 & 78,060 & $1,244,962$ & 215,126 & $2,784,270$ & Mean \\
\hline 650,251 & 315,951 & 61,655 & $1,042,197$ & 164,568 & $2,466,438$ & Median \\
\hline 127,905 & 88,527 & 9,025 & 308,055 & 6,466 & $1,044,537$ & Low \\
\hline $54,707,537$ & $30,863,167$ & $5,776,460$ & $93,372,148$ & $15,704,180$ & $208,820,262$ & Sum \\
\hline 74 & 74 & 74 & 75 & 73 & 75 & Number of Libraries \\
\hline
\end{tabular}




\section{Electronic Resources Expenditures}

One-time Electronic Ongoing Electronic Resource Purchases Resource Purchases
Total Electron

Resource Purchases
Electronic Resources as a \% of Library Materials Budget

(Survey Question Number)

(21)

(22)

$(21+22)$

(15)

\begin{tabular}{|c|c|c|c|c|c|c|}
\hline INSTITUTION & Notes & & & & & \\
\hline ALABAMA & bG+ & 0 & 206,181 & 206,181 & $1,369,026$ & 15 \\
\hline ARIZONA & G+ & UA/NA & $\mathrm{UA} / \mathrm{NA}$ & 0 & $1,101,576$ & 0 \\
\hline ARIZONA STATE & G & 0 & 288,328 & 288,328 & 954,065 & 30 \\
\hline BOSTON & + & 12,000 & 214,655 & 226,655 & $1,235,316$ & 18 \\
\hline BOSTON COLLEGE & bG+ & UA/NA & 85,533 & 85,533 & $1,274,889$ & 7 \\
\hline BRIGHAM YOUNG & bG+ & 62,000 & 255,068 & 317,068 & 822,836 & 39 \\
\hline CALIFORNIA, BERKELEY & G & 0 & 0 & 0 & $2,499,620$ & UA/NA \\
\hline CALIFORNIA, DAVIS & G+ & 0 & 179,313 & 179,313 & 968,139 & 19 \\
\hline CALIFORNIA, LOS ANGELES & G+ & UA/NA & $\mathrm{UA} / \mathrm{NA}$ & 0 & $1,827,051$ & UA/NA \\
\hline CASE WESTERN RESERVE & G+ & 0 & 206,705 & 206,705 & $1,328,670$ & 16 \\
\hline CHICAGO & G+ & 43,000 & 181,438 & 224,438 & $1,986,134$ & 11 \\
\hline CINCINNATI & G+ & 0 & 94,838 & 94,838 & 873,121 & 11 \\
\hline COLORADO & G & 101,595 & 143,929 & 245,524 & $1,318,406$ & 19 \\
\hline COLUMBIA & bG+ & 0 & 359,720 & 359,720 & $2,192,600$ & 16 \\
\hline CONNECTICUT & G & 0 & 181,468 & 181,468 & $1,455,182$ & 12 \\
\hline CORNELL & bG+ & 0 & 91,323 & 91,323 & $1,447,560$ & 6 \\
\hline DUKE & + & UA/NA & 204,091 & 204,091 & $1,708,420$ & 12 \\
\hline EMORY & + & 0 & 235,332 & 235,332 & $1,150,104$ & 20 \\
\hline FLORIDA & G+ & 87,048 & 426,350 & 513,398 & $1,253,347$ & 41 \\
\hline FLORIDA STATE & G+ & 0 & 279,356 & 279,356 & 877,776 & 32 \\
\hline GEORGE WASHINGTON & bG+ & 57,850 & 369,562 & 427,412 & $2,790,982$ & 15 \\
\hline GEORGETOWN & bG+ & 176,086 & 313,590 & 489,676 & $2,773,803$ & 18 \\
\hline GEORGIA & + & 405,743 & 151,204 & 556,947 & $1,447,831$ & 38 \\
\hline HARVARD & + & 372,874 & UA/NA & 372,874 & $3,733,674$ & 10 \\
\hline HAWAII & G & 0 & 134,999 & 134,999 & 656,959 & 21 \\
\hline HOUSTON & bG+ & 0 & 240,447 & 240,447 & 981,717 & 24 \\
\hline HOWARD & bG+ & 0 & 92,118 & 92,118 & 577,400 & 16 \\
\hline ILLINOIS, URBANA & bG+ & 0 & 147,573 & 147,573 & $1,006,761$ & 15 \\
\hline INDIANA & G+ & 750 & 303,462 & 304,212 & $1,531,852$ & 20 \\
\hline IOWA & G+ & 76,908 & 164,546 & 241,454 & $2,652,699$ & 9 \\
\hline KANSAS & + & UA/NA & $\mathrm{UA} / \mathrm{NA}$ & 0 & 557,903 & UA/NA \\
\hline KENTUCKY & G & 0 & 100,880 & 100,880 & $1,078,439$ & 9 \\
\hline LOUISIANA STATE & G+ & 0 & 229,723 & 229,723 & $1,199,816$ & 19 \\
\hline LOUISVILLE & G & UA/NA & 90,514 & 90,514 & $1,334,393$ & 7 \\
\hline MIAMI & G+ & 39,000 & 318,162 & 357,162 & $2,288,314$ & 16 \\
\hline MICHIGAN & G+ & 35,000 & 210,150 & 245,150 & $2,449,375$ & 10 \\
\hline MICHIGAN STATE & bG+ & $\mathrm{UA} / \mathrm{NA}$ & 326,418 & 326,418 & 991,468 & 33 \\
\hline MINNESOTA & bG+ & 58,333 & 218,298 & 276,631 & $1,718,995$ & 16 \\
\hline MISSOURI & bG+ & UA/NA & 234,785 & 234,785 & 533,968 & 44 \\
\hline MONTREAL & G+ & UA/NA & 118,592 & 118,592 & 700,479 & 17 \\
\hline NEBRASKA & G+ & 0 & 194,375 & 194,375 & 571,984 & 34 \\
\hline NEW MEXICO & G & 105,833 & UA/NA & 105,833 & 408,844 & 26 \\
\hline
\end{tabular}




\section{Electronic Resources Expenditures}

Bibl. Utilities, Networks, etc. Bibl. Utilities, Networks, etc. Library Expenditures

\section{External Expeditures}

Computer Hardware and Software Expenditures

Document Delivery/

Interlibrary Loan Expenditures
(23a)
(23b)
(24)
(25) (Survey Question Number)

INSTITUTION

\begin{tabular}{|c|c|c|c|c|}
\hline 46,768 & 0 & 31,775 & 823 & ALABAMA \\
\hline $\mathrm{UA} / \mathrm{NA}$ & $\mathrm{UA} / \mathrm{NA}$ & 9,971 & 102 & ARIZONA \\
\hline 12,984 & 0 & 8,078 & 1,081 & ARIZONA STATE \\
\hline 34,875 & 0 & 3,999 & 5,725 & BOSTON \\
\hline 18,411 & 0 & 36,719 & 231 & BOSTON COLLEGE \\
\hline 0 & $\mathrm{UA} / \mathrm{NA}$ & 1,565 & UA/NA & BRIGHAM YOUNG \\
\hline 0 & 0 & 0 & 0 & CALIFORNIA, BERKELEY \\
\hline 16,919 & 0 & 0 & 35 & CALIFORNIA, DAVIS \\
\hline $\mathrm{UA} / \mathrm{NA}$ & $\mathrm{UA} / \mathrm{NA}$ & $\mathrm{UA} / \mathrm{NA}$ & UA/NA & CALIFORNIA, LOS ANGELES \\
\hline 6,212 & 0 & 0 & 217 & CASE WESTERN RESERVE \\
\hline 30,446 & 0 & 48,732 & 804 & CHICAGO \\
\hline 6,272 & 0 & 56,810 & 1,470 & CINCINNATI \\
\hline 22,000 & 0 & 28,609 & 323 & COLORADO \\
\hline 6,834 & 0 & 111,684 & 19,523 & COLUMBIA \\
\hline 28,000 & 0 & 49,178 & 12,192 & CONNECTICUT \\
\hline 0 & 0 & 4,566 & 5,303 & CORNELL \\
\hline 10,000 & 0 & 17,940 & 419 & DUKE \\
\hline 13,829 & 0 & 1,156 & 2,819 & EMORY \\
\hline 0 & 6,427 & 9,347 & 76 & FLORIDA \\
\hline 0 & 47,731 & 17,058 & 1,073 & FLORIDA STATE \\
\hline 0 & 35,663 & 97,298 & 45,822 & GEORGE WASHINGTON \\
\hline 50,727 & $\mathrm{UA} / \mathrm{NA}$ & 51,775 & 14,985 & GEORGETOWN \\
\hline 62,207 & 26,550 & 21,566 & 659 & GEORGIA \\
\hline 23,632 & $\mathrm{UA} / \mathrm{NA}$ & 161,784 & UA/NA & HARVARD \\
\hline 45,263 & $\mathrm{UA} / \mathrm{NA}$ & 8,665 & 0 & HAWAII \\
\hline 31,997 & 0 & 2,440 & 1,944 & HOUSTON \\
\hline 0 & 0 & 11,932 & 125 & HOWARD \\
\hline $\mathrm{UA} / \mathrm{NA}$ & $\mathrm{UA} / \mathrm{NA}$ & 5,000 & $\mathrm{UA} / \mathrm{NA}$ & ILLINOIS, URBANA \\
\hline 6,890 & 0 & 0 & 0 & INDIANA \\
\hline 21,965 & $\mathrm{UA} / \mathrm{NA}$ & 76,480 & 8,053 & IOWA \\
\hline $\mathrm{UA} / \mathrm{NA}$ & 7,400 & 21,548 & 46 & KANSAS \\
\hline 5,000 & 0 & 4,346 & 440 & KENTUCKY \\
\hline 32,710 & 20,277 & 58,845 & 1,404 & LOUISIANA STATE \\
\hline $\mathrm{UA} / \mathrm{NA}$ & $\mathrm{UA} / \mathrm{NA}$ & $\mathrm{UA} / \mathrm{NA}$ & 519 & LOUISVILLE \\
\hline 20,000 & 0 & 53,568 & 456 & MIAMI \\
\hline 57,024 & 0 & 131,676 & 10,317 & MICHIGAN \\
\hline 14,477 & 0 & 0 & 3,323 & MICHIGAN STATE \\
\hline 15,483 & 0 & 8,174 & 2,199 & MINNESOTA \\
\hline 21,241 & $\mathrm{UA} / \mathrm{NA}$ & 13,199 & 926 & MISSOURI \\
\hline UA/NA & UA/NA & UA/NA & UA/NA & MONTREAL \\
\hline 0 & 4,997 & 39,395 & 0 & NEBRASKA \\
\hline $\mathrm{UA} / \mathrm{NA}$ & UA/NA & 33,559 & UA/NA & NEW MEXICO \\
\hline
\end{tabular}




\section{Electronic Resources Expenditures}

One-time Electronic Resource Purchases
Ongoing Electronic

Resource Purchases
Total Electron

Resource Purchases
Electronic Resources

as a \% of Library Materials Budget

(Survey Question Number)

(21)

(22)

$(21+22)$

(15)

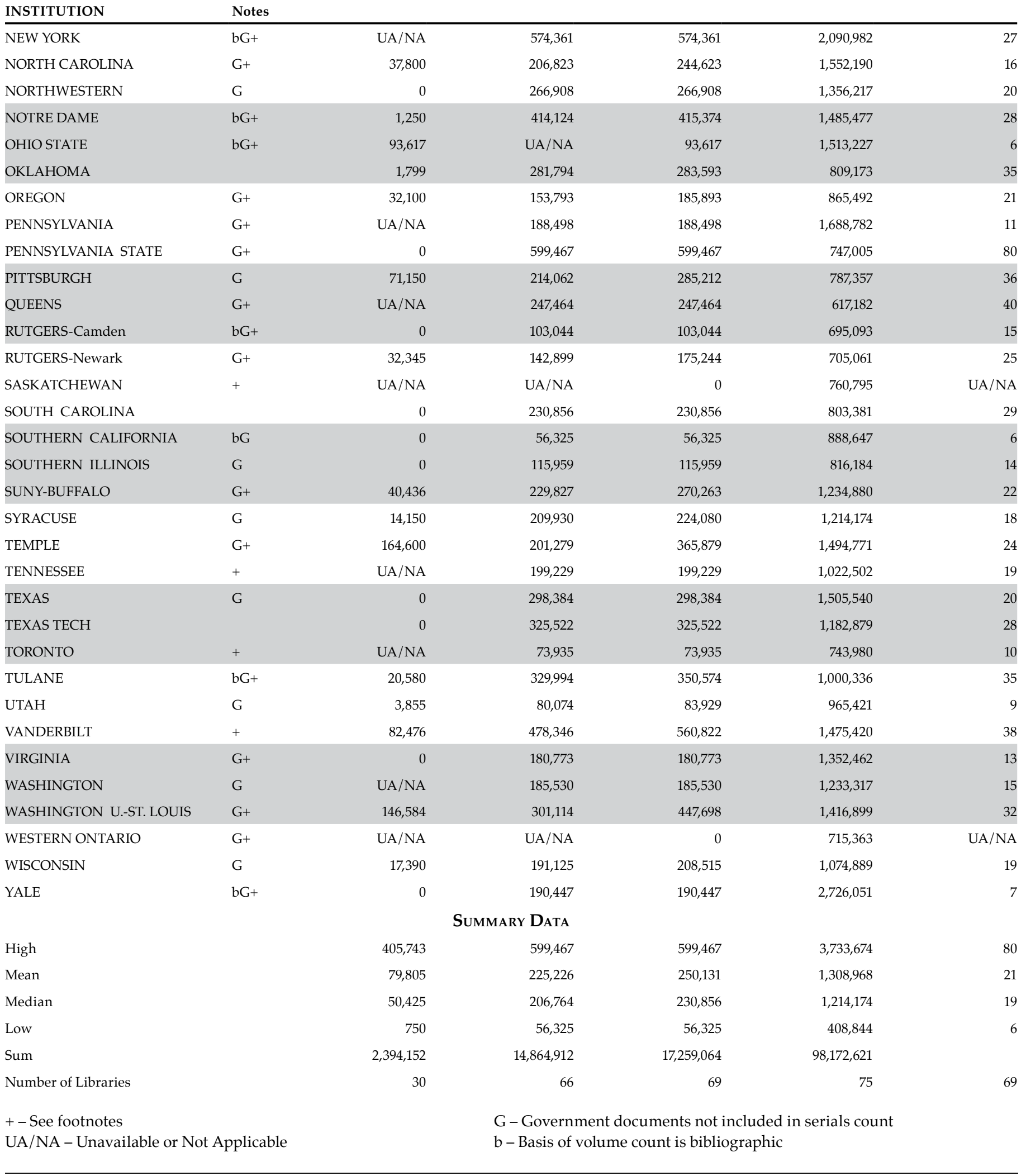




\section{Electronic Resources Expenditures}

Bibl. Utilities, Networks, etc. Bibl. Utilities, Networks, etc. Library Expenditures
Computer Hardware and Software Expenditures

Document Delivery/

Interlibrary Loan Expenditures

(23a) (23b)
(24)
(25) (Survey Question Number)

\begin{tabular}{|c|c|c|c|c|}
\hline 40,000 & UA/NA & 84,000 & $\mathrm{UA} / \mathrm{NA}$ & NEW YORK \\
\hline 10,000 & UA/NA & 4,598 & 414 & NORTH CAROLINA \\
\hline 18,173 & 0 & 31,732 & 5,223 & NORTHWESTERN \\
\hline 0 & 33,369 & 11,963 & 4,371 & NOTRE DAME \\
\hline 25,000 & UA/NA & 12,488 & 137 & OHIO STATE \\
\hline 12,192 & 0 & 10,452 & 0 & OKLAHOMA \\
\hline 5,649 & 0 & 34,449 & $\mathrm{UA} / \mathrm{NA}$ & OREGON \\
\hline 33,491 & UA/NA & 58,457 & $\mathrm{UA} / \mathrm{NA}$ & PENNSYLVANIA \\
\hline 0 & 22,018 & 17,481 & 140 & PENNSYLVANIA STATE \\
\hline $\mathrm{UA} / \mathrm{NA}$ & 6,846 & 4,440 & 253 & PITTSBURGH \\
\hline UA/NA & UA/NA & UA/NA & $\mathrm{UA} / \mathrm{NA}$ & QUEENS \\
\hline 9,172 & 0 & 961 & 2,500 & RUTGERS \\
\hline 18,900 & 0 & 46,688 & 3,251 & RUTGERS \\
\hline $\mathrm{UA} / \mathrm{NA}$ & UA/NA & $\mathrm{UA} / \mathrm{NA}$ & $\mathrm{UA} / \mathrm{NA}$ & SASKATCHEWAN \\
\hline 7,968 & 0 & 0 & 1,295 & SOUTH CAROLINA \\
\hline 5,423 & 4,117 & 3,715 & 3,428 & SOUTHERN CALIFORNIA \\
\hline 2,170 & 0 & 0 & 0 & SOUTHERN ILLINOIS \\
\hline UA/NA & UA/NA & UA/NA & $\mathrm{UA} / \mathrm{NA}$ & SUNY-BUFFALO \\
\hline 22,156 & 0 & 5,792 & 1,764 & SYRACUSE \\
\hline 2,232 & 0 & 132,530 & 4,231 & TEMPLE \\
\hline 17,255 & UA/NA & 24,350 & 176 & TENNESSEE \\
\hline 11,742 & 0 & 142,427 & 478 & TEXAS \\
\hline 12,762 & 0 & 228,609 & 995 & TEXAS TECH \\
\hline $\mathrm{UA} / \mathrm{NA}$ & UA/NA & 68,445 & $\mathrm{UA} / \mathrm{NA}$ & TORONTO \\
\hline 10,746 & 0 & 78,610 & 7,003 & TULANE \\
\hline 21,729 & 3,000 & 12,074 & 674 & UTAH \\
\hline 6,406 & 0 & 39,655 & 1,000 & VANDERBILT \\
\hline 20,000 & 1,997 & 44,944 & 19,727 & VIRGINIA \\
\hline 20,684 & UA/NA & 38,412 & $\mathrm{UA} / \mathrm{NA}$ & WASHINGTON \\
\hline 30,997 & 0 & 80,174 & 2,383 & WASHINGTON U.-ST. LOUIS \\
\hline UA/NA & 0 & UA/NA & 0 & WESTERN ONTARIO \\
\hline $\mathrm{UA} / \mathrm{NA}$ & UA/NA & 14,527 & 329 & WISCONSIN \\
\hline 0 & 275,000 & 60,000 & 11,000 & YALE \\
\hline \multicolumn{5}{|c|}{ Summary Data } \\
\hline 62,207 & 275,000 & 228,609 & 45,822 & High \\
\hline 20,540 & 35,385 & 41,482 & 3,967 & Mean \\
\hline 18,292 & 13,839 & 28,609 & 1,077 & Median \\
\hline 2,170 & 1,997 & 961 & 35 & Low \\
\hline $1,027,013$ & 495,392 & $2,530,410$ & 214,206 & Sum \\
\hline 50 & 14 & 61 & 54 & Number of Libraries \\
\hline
\end{tabular}


Personnel and Public Services

Professional Staff Support Staff (FTE) Student Assistants (FTE)

Total Staff (FTE)

(26a)

(26b)

(26c)

(26)
Staffed Service

Points

(27)

Library Service

Hours

(Survey Question Number)

INSTITUTION

\begin{tabular}{|c|c|c|c|c|c|c|c|}
\hline ALABAMA & bG+ & 9 & 7 & 5 & 21 & 4 & 110 \\
\hline ARIZONA & G+ & 13 & 12 & 1 & 26 & 2 & 99 \\
\hline ARIZONA STATE & G & 9 & 13 & 2 & 24 & 2 & 111 \\
\hline BOSTON & + & 11 & 14 & 6 & 31 & 3 & 102 \\
\hline BOSTON COLLEGE & bG+ & 14 & 10 & 5 & 29 & 2 & 105 \\
\hline BRIGHAM YOUNG & bG+ & 11 & 4 & 19 & 34 & 3 & 105 \\
\hline CALIFORNIA, BERKELEY & G & 18 & 25 & 14 & 57 & 0 & $\mathrm{UA} / \mathrm{NA}$ \\
\hline CALIFORNIA, DAVIS & G+ & 7 & 10 & 2 & 19 & 2 & 78 \\
\hline CALIFORNIA, LOS ANGELES & G+ & 14 & 15 & 5 & 34 & 1 & $\mathrm{UA} / \mathrm{NA}$ \\
\hline CASE WESTERN RESERVE & G+ & 13 & 10 & 5 & 28 & 2 & $\mathrm{UA} / \mathrm{NA}$ \\
\hline CHICAGO & G+ & 10 & 17 & 3 & 30 & 2 & 90 \\
\hline CINCINNATI & G+ & 8 & 6 & 3 & 17 & 2 & 95 \\
\hline COLORADO & G & 8 & 10 & 2 & 20 & 2 & 104 \\
\hline COLUMBIA & $\mathrm{bG}+$ & 20 & 24 & 5 & 49 & 2 & -1 \\
\hline CONNECTICUT & G & 12 & 11 & 3 & 26 & 3 & 89 \\
\hline CORNELL & bG+ & 10 & 12 & 4 & 26 & 2 & 80 \\
\hline DUKE & + & 11 & 11 & 4 & 26 & 3 & 104 \\
\hline EMORY & + & 10 & 7 & 4 & 21 & 2 & 109 \\
\hline FLORIDA & G+ & 10 & 11 & 19 & 40 & 2 & 94 \\
\hline FLORIDA STATE & G+ & 10 & 7 & 5 & 22 & 6 & $\mathrm{UA} / \mathrm{NA}$ \\
\hline GEORGE WASHINGTON & $\mathrm{bG}^{+}$ & 20 & 21 & 6 & 47 & 3 & 110 \\
\hline GEORGETOWN & bG+ + & 27 & 41 & 18 & 86 & 5 & 107 \\
\hline GEORGIA & + & 8 & 11 & 4 & 23 & 3 & 118 \\
\hline HARVARD & + & 48 & 70 & 5 & 123 & UA/NA & $\mathrm{UA} / \mathrm{NA}$ \\
\hline HAWAII & G & 5 & 4 & 6 & 15 & 2 & 94 \\
\hline HOUSTON & bG+ & 13 & 9 & 7 & 29 & 3 & -1 \\
\hline HOWARD & bG+ & 10 & 10 & 10 & 30 & 2 & 115 \\
\hline ILLINOIS, URBANA & bG+ & 12 & 6 & 4 & 22 & 2 & 102 \\
\hline INDIANA & G+ & 11 & 9 & 4 & 24 & 4 & 115 \\
\hline IOWA & G+ & 16 & 17 & 6 & 39 & 3 & 106 \\
\hline KANSAS & + & 7 & 6 & 8 & 21 & 2 & 95 \\
\hline KENTUCKY & G & 7 & 6 & 3 & 16 & 2 & 135 \\
\hline LOUISIANA STATE & G+ & 11 & 10 & 15 & 36 & 3 & 101 \\
\hline LOUISVILLE & G & 6 & 8 & 2 & 16 & 1 & 89 \\
\hline MIAMI & G+ & 14 & 15 & 5 & 34 & 2 & 111 \\
\hline MICHIGAN & G+ & 11 & 29 & 13 & 53 & 2 & 112 \\
\hline MICHIGAN STATE & bG+ & 9 & 4 & 7 & 20 & 3 & 109 \\
\hline MINNESOTA & bG+ & 14 & 13 & 9 & 36 & 3 & 81 \\
\hline MISSOURI & bG+ & 8 & 9 & 4 & 21 & 5 & 73 \\
\hline MONTREAL & G+ & 5 & 11 & 0 & 16 & 3 & 89 \\
\hline NEBRASKA & G+ & 5 & 8 & 4 & 17 & 2 & 109 \\
\hline NEW MEXICO & G & 7 & 10 & 3 & 20 & 2 & 90 \\
\hline
\end{tabular}




\section{Personnel and Public Services}

\begin{tabular}{|c|c|c|c|c|c|c|c|}
\hline $\begin{array}{r}\text { Library } \\
\text { Presentations to } \\
\text { Groups }\end{array}$ & $\begin{array}{r}\text { Participants } \\
\text { in Group } \\
\text { Presentations }\end{array}$ & $\begin{array}{r}\text { Reference } \\
\text { Transactions }\end{array}$ & $\begin{array}{r}\text { Initial } \\
\text { Circulation } \\
\text { Transactions }\end{array}$ & $\begin{array}{r}\text { Total } \\
\text { Circulation } \\
\text { Transactions }\end{array}$ & $\begin{array}{r}\text { Total Items } \\
\text { Loaned (ILL) }\end{array}$ & $\begin{array}{r}\text { Total Items } \\
\text { Borrowed (ILL) }\end{array}$ & \\
\hline (29) & (30) & (31) & (32) & (33) & (34) & (35) & (Survey Question Number) \\
\hline
\end{tabular}
(29)
(31)
$(32)$

\begin{tabular}{|c|c|c|c|c|c|c|c|}
\hline 50 & UA/NA & 2,282 & 5,751 & 7,012 & 438 & 163 & ALABAMA \\
\hline 591 & 4,354 & 3,676 & 3,526 & 4,643 & 265 & 172 & ARIZONA \\
\hline 35 & 835 & 13,793 & 3,682 & 26,204 & 654 & 614 & ARIZONA STATE \\
\hline 293 & 3,967 & 6,275 & 6,863 & 14,318 & 187 & 1,465 & BOSTON \\
\hline 310 & 7,184 & 1,474 & 5,942 & 8,641 & 638 & 705 & BOSTON COLLEGE \\
\hline 263 & 5,656 & 7,662 & 8,739 & 13,010 & 671 & 579 & BRIGHAM YOUNG \\
\hline 0 & 0 & 0 & 15,849 & 33,280 & 92 & 144 & CALIFORNIA, BERKELEY \\
\hline 82 & 1,302 & 16,120 & 11,651 & 14,905 & 171 & 569 & CALIFORNIA, DAVIS \\
\hline 103 & 2,282 & 4,247 & 10,993 & 285,025 & $\mathrm{UA} / \mathrm{NA}$ & UA/NA & CALIFORNIA, LOS ANGELES \\
\hline 79 & 1,411 & 8,100 & 5,021 & 10,255 & 686 & 1,844 & CASE WESTERN RESERVE \\
\hline 128 & 1,774 & 5,700 & 19,722 & 24,405 & 39 & 694 & CHICAGO \\
\hline 47 & 181 & 13,445 & 4,904 & 7,982 & 102 & 94 & CINCINNATI \\
\hline 78 & 317 & 2,454 & 6,388 & 8,656 & 1,060 & 948 & COLORADO \\
\hline 121 & 1,858 & 14,112 & 17,740 & 25,845 & 6,085 & 686 & COLUMBIA \\
\hline 101 & 1,202 & 4,946 & 5,621 & 8,815 & 578 & 1,669 & CONNECTICUT \\
\hline 237 & 1,201 & 4,641 & 13,984 & 18,418 & 1,742 & 1,146 & CORNELL \\
\hline 80 & 808 & 4,750 & 27,889 & 42,930 & 530 & 847 & DUKE \\
\hline 53 & 457 & 4,734 & 6,137 & 7,680 & 452 & 1,020 & EMORY \\
\hline UA/NA & UA/NA & UA/NA & 7,779 & 13,441 & 96 & 434 & FLORIDA \\
\hline 119 & 1,917 & 5,304 & 3,297 & 6,743 & 336 & 378 & FLORIDA STATE \\
\hline 54 & 830 & UA/NA & 6,182 & 9,537 & 1,676 & 1,501 & GEORGE WASHINGTON \\
\hline 334 & 4,890 & 10,906 & 16,222 & 36,983 & 3,477 & 2,233 & GEORGETOWN \\
\hline 26 & 697 & 3,550 & 4,448 & 8,678 & 777 & 150 & GEORGIA \\
\hline UA/NA & UA/NA & UA/NA & UA/NA & 62,289 & 1,561 & 1,190 & HARVARD \\
\hline 37 & 852 & 2,853 & 3,016 & 4,478 & 372 & 16 & HAWAII \\
\hline 79 & 1,325 & 3,661 & 3,390 & 5,670 & 337 & 833 & HOUSTON \\
\hline 100 & 1,200 & 1,900 & 1,012 & 1,658 & 1,200 & 110 & HOWARD \\
\hline 106 & 305 & 2,315 & $\mathrm{UA} / \mathrm{NA}$ & UA/NA & $\mathrm{UA} / \mathrm{NA}$ & UA/NA & ILLINOIS, URBANA \\
\hline 122 & 2,828 & 20,300 & 18,578 & 25,515 & 1,341 & 643 & INDIANA \\
\hline 224 & 2,902 & 9,078 & 20,745 & 25,154 & 1,413 & 837 & IOWA \\
\hline 42 & 380 & 6,984 & 9,750 & 13,750 & 820 & 403 & KANSAS \\
\hline 7 & 89 & 1,796 & 5,447 & 6,970 & 227 & 470 & KENTUCKY \\
\hline 37 & 958 & 4,471 & 2,784 & 4,088 & 231 & 196 & LOUISIANA STATE \\
\hline UA/NA & UA/NA & UA/NA & $\mathrm{UA} / \mathrm{NA}$ & UA/NA & $\mathrm{UA} / \mathrm{NA}$ & UA/NA & LOUISVILLE \\
\hline 383 & 7,397 & 29,004 & 7,509 & 19,852 & 631 & 714 & MIAMI \\
\hline 74 & 1,787 & 6,306 & 30,760 & 54,826 & 1,043 & 480 & MICHIGAN \\
\hline 85 & 2,642 & 5,667 & 4,694 & 10,950 & 263 & 545 & MICHIGAN STATE \\
\hline 180 & 1,070 & 9,200 & 16,121 & 23,641 & 2,611 & 765 & MINNESOTA \\
\hline 150 & 1,500 & 2,300 & 3,572 & 4,614 & 1,451 & 360 & MISSOURI \\
\hline 73 & 1,227 & 12,887 & 24,360 & 40,640 & 494 & 348 & MONTREAL \\
\hline 20 & 539 & 4,149 & 45,182 & 59,278 & 648 & 184 & NEBRASKA \\
\hline 120 & 1,170 & 3,782 & 3,514 & 9,753 & 368 & 128 & NEW MEXICO \\
\hline
\end{tabular}


Personnel and Public Services

Professional Staff

(FTE)

(Survey Question Number)
Support Staff Student Assistants (FTE)
(FTE)
Total Staff

(FTE)
Staffed Service

Points
Library Service

Hours
(26b)
(26c)
(26a)
(26)
(27)

(28)

INSTITUTION

Notes

\begin{tabular}{|c|c|c|c|c|c|c|c|}
\hline NEW YORK & bG+ & 13 & 30 & 20 & 63 & 6 & 91 \\
\hline NORTH CAROLINA & G+ & 13 & 9 & 4 & 26 & 2 & 109 \\
\hline NORTHWESTERN & G & 11 & 18 & 2 & 31 & 4 & 105 \\
\hline NOTRE DAME & bG+ & 10 & 13 & 9 & 32 & 2 & 95 \\
\hline OHIO STATE & $\mathrm{bG}+$ & 7 & 10 & 5 & 22 & 2 & 105 \\
\hline OKLAHOMA & & 7 & 7 & 8 & 22 & 2 & 96 \\
\hline OREGON & G+ & 7 & 6 & 5 & 18 & 2 & 106 \\
\hline PENNSYLVANIA & G+ & 15 & 19 & 2 & 36 & 2 & 116 \\
\hline PENNSYLVANIA STATE & G+ & 10 & 12 & 6 & 28 & 2 & 94 \\
\hline PITTSBURGH & G & 8 & 10 & 2 & 20 & 1 & 103 \\
\hline QUEENS & G+ & 3 & 7 & 2 & 12 & 2 & 95 \\
\hline RUTGERS-Camden & $\mathrm{bG}+$ & 7 & 12 & 2 & 21 & 2 & 103 \\
\hline RUTGERS-Newark & $\mathrm{G}+$ & 9 & 10 & 2 & 21 & 3 & 95 \\
\hline SASKATCHEWAN & + & 3 & 5 & 1 & 9 & 27 & 85 \\
\hline SOUTH CAROLINA & & 8 & 7 & 2 & 17 & 2 & 107 \\
\hline SOUTHERN CALIFORNIA & bG & 10 & 9 & 4 & 23 & 3 & 100 \\
\hline SOUTHERN ILLINOIS & G & 4 & 8 & 8 & 20 & 3 & 78 \\
\hline SUNY-BUFFALO & $\mathrm{G}+$ & 12 & 10 & 8 & 30 & 3 & 105 \\
\hline SYRACUSE & G & 12 & 9 & 5 & 26 & 2 & 106 \\
\hline TEMPLE & G+ & 12 & 7 & 6 & 25 & 2 & 96 \\
\hline TENNESSEE & + & 9 & 13 & 11 & 33 & 2 & -1 \\
\hline TEXAS & G & 15 & 21 & 4 & 40 & 5 & 99 \\
\hline TEXAS TECH & & 8 & 19 & 5 & 32 & 3 & 96 \\
\hline TORONTO & + & 6 & 7 & 4 & 17 & 2 & 88 \\
\hline TULANE & $\mathrm{bG}+$ & 8 & 9 & 4 & 21 & 3 & 112 \\
\hline UTAH & G & 8 & 9 & 5 & 22 & 4 & 95 \\
\hline VANDERBILT & + & 6 & 9 & 5 & 20 & 2 & 111 \\
\hline VIRGINIA & G+ & 12 & 17 & 3 & 32 & 7 & -1 \\
\hline WASHINGTON & G & 14 & 14 & 5 & 33 & 3 & 89 \\
\hline WASHINGTON U.-ST. LOUIS & G+ & 9 & 11 & 6 & 26 & 2 & 120 \\
\hline WESTERN ONTARIO & G+ & 4 & 4 & 1 & 9 & 1 & 80 \\
\hline WISCONSIN & G & 12 & 11 & 8 & 31 & 3 & 104 \\
\hline YALE & $\mathrm{bG}+$ & 20 & 24 & 15 & 59 & 5 & 86 \\
\hline \multicolumn{8}{|c|}{ Summary Data } \\
\hline \multicolumn{2}{|l|}{ High } & 48 & 70 & 20 & 123 & 27 & 135 \\
\hline \multicolumn{2}{|l|}{ Mean } & 11 & 13 & 6 & 29 & 3 & 100 \\
\hline \multicolumn{2}{|l|}{ Median } & 10 & 10 & 5 & 26 & 2 & 102 \\
\hline \multicolumn{2}{|l|}{ Low } & 3 & 4 & 1 & 9 & 1 & 73 \\
\hline \multicolumn{2}{|l|}{ Sum } & 814 & 949 & 438 & 2,201 & 220 & \\
\hline \multicolumn{2}{|l|}{ Number of Libraries } & 75 & 75 & 74 & 75 & 73 & 6 \\
\hline \multicolumn{3}{|c|}{$\begin{array}{l}+- \text { See footnotes } \\
\text { UA/NA - Unavailable or Not Applicable }\end{array}$} & \multicolumn{4}{|c|}{$\begin{array}{l}\text { G- Government documents not included in serials count } \\
b-\text { Basis of volume count is bibliographic }\end{array}$} & \\
\hline
\end{tabular}




\section{Personnel and Public Services}

\begin{tabular}{|c|c|c|c|c|c|c|}
\hline $\begin{array}{r}\text { Library } \\
\text { Presentations to } \\
\text { Groups * }\end{array}$ & $\begin{array}{r}\text { Participants } \\
\text { in Group } \\
\text { Presentations * }\end{array}$ & $\begin{array}{r}\text { Reference } \\
\text { Transactions * }\end{array}$ & $\begin{array}{r}\text { Initial } \\
\text { Circulation } \\
\text { Transactions }\end{array}$ & $\begin{array}{r}\text { Total } \\
\text { Circulation } \\
\text { Transactions }\end{array}$ & $\begin{array}{r}\text { Total Items } \\
\text { Loaned (ILL) }\end{array}$ & $\begin{array}{r}\text { Total Items } \\
\text { Borrowed (ILL) }\end{array}$ \\
\hline
\end{tabular}
(29)
(30)
(31)
(32)
(33)
(34)
(35) (Survey Question Number)

\begin{tabular}{|c|c|c|c|c|c|c|c|}
\hline 80 & 1,300 & 19,000 & 18,500 & 64,000 & 970 & 1,850 & $\overline{\text { NEW YORK }}$ \\
\hline 166 & 3,238 & 5,295 & 3,742 & 7,728 & 548 & 357 & NORTH CAROLINA \\
\hline 118 & 1,197 & 5,432 & 9,794 & 17,294 & 1,732 & 1,800 & NORTHWESTERN \\
\hline $\mathrm{UA} / \mathrm{NA}$ & UA/NA & UA/NA & 11,438 & 19,427 & 932 & 848 & NOTRE DAME \\
\hline 174 & 987 & 4,877 & 9,847 & 41,572 & 336 & 289 & OHIO STATE \\
\hline 344 & 1,115 & 1,581 & 3,832 & 5,142 & 103 & 337 & OKLAHOMA \\
\hline 59 & 579 & 2,029 & 4,425 & 7,852 & UA/NA & $\mathrm{UA} / \mathrm{NA}$ & OREGON \\
\hline 281 & 4,737 & UA/NA & 36,612 & 38,655 & 1,312 & 1,238 & PENNSYLVANIA \\
\hline 67 & 953 & 3,280 & 3,481 & 4,223 & 9 & 419 & PENNSYLVANIA STATE \\
\hline 89 & 1,572 & 2,984 & 2,958 & 4,492 & 402 & 100 & PITTSBURGH \\
\hline 19 & 1,530 & 1,956 & 7,218 & 8,108 & UA/NA & $\mathrm{UA} / \mathrm{NA}$ & QUEENS \\
\hline 6 & 180 & 1,341 & 4,015 & 4,064 & 71 & 266 & RUTGERS \\
\hline 26 & 450 & 11,735 & 3,013 & 6,627 & 23 & 154 & RUTGERS \\
\hline 34 & 1,212 & 2,452 & 12,651 & 15,935 & 255 & 48 & SASKATCHEWAN \\
\hline 450 & 355 & 5,250 & 6,883 & 6,883 & 100 & 492 & SOUTH CAROLINA \\
\hline 365 & 420 & 2,871 & 5,820 & 12,286 & 81 & 488 & SOUTHERN CALIFORNIA \\
\hline 121 & 334 & 1,879 & 2,653 & 3,454 & 433 & 140 & SOUTHERN ILLINOIS \\
\hline 80 & 980 & 9,100 & 7,491 & 13,051 & 892 & 900 & SUNY-BUFFALO \\
\hline 84 & 1,213 & 10,594 & 4,227 & 7,496 & 252 & 389 & SYRACUSE \\
\hline 93 & 1,251 & 23,482 & 5,694 & 6,604 & 18 & 339 & TEMPLE \\
\hline $\mathrm{UA} / \mathrm{NA}$ & UA/NA & UA/NA & UA/NA & $\mathrm{UA} / \mathrm{NA}$ & 80 & 110 & TENNESSEE \\
\hline 24 & 432 & 5,943 & 19,113 & 39,147 & 1,458 & 588 & TEXAS \\
\hline 76 & 1,355 & 3,896 & 16,470 & 17,167 & 323 & 270 & TEXAS TECH \\
\hline 184 & 1,507 & 39,019 & 11,036 & 16,498 & 744 & 255 & TORONTO \\
\hline 46 & 1,010 & 5,280 & 5,818 & 6,421 & 257 & 954 & TULANE \\
\hline 207 & 1,154 & 8,424 & 7,180 & 15,322 & 850 & 429 & UTAH \\
\hline 113 & 226 & 2,730 & 4,043 & 8,138 & 453 & 3,350 & VANDERBILT \\
\hline 28 & 400 & 13,500 & 36,978 & 46,073 & 1,183 & 776 & VIRGINIA \\
\hline 164 & 2,551 & UA/NA & UA/NA & 21,060 & 1,615 & 1,956 & WASHINGTON \\
\hline 257 & 940 & UA/NA & 8,283 & 17,624 & 868 & 1,297 & WASHINGTON U.-ST. LOUIS \\
\hline 66 & 605 & 2,240 & 8,844 & 10,549 & 428 & 0 & WESTERN ONTARIO \\
\hline 52 & 632 & UA/NA & 8,669 & 14,036 & 380 & 620 & WISCONSIN \\
\hline 140 & 1,330 & 6,553 & 27,993 & 47,237 & 1,679 & 2,300 & YALE \\
\hline \multicolumn{8}{|c|}{ SUMMARY DATA } \\
\hline 591 & 7,397 & 39,019 & 45,182 & 285,025 & 6,085 & 3,350 & High \\
\hline 131 & 1,574 & 7,305 & 10,536 & 21,732 & 779 & 719 & Mean \\
\hline 89 & 1,199 & 5,098 & 7,032 & 13,031 & 512 & 545 & Median \\
\hline 6 & 89 & 1,341 & 1,012 & 1,658 & 9 & 16 & Low \\
\hline 9,036 & 107,039 & 467,547 & 737,485 & $1,564,697$ & 54,550 & 49,636 & Sum \\
\hline 69 & 68 & 64 & 70 & 72 & 70 & 69 & Number of Libraries \\
\hline
\end{tabular}

* Figures in italics are derived from a sampling method rather than an actual annual count. 
Rank Order Table 1: Volumes in Library

\begin{tabular}{|c|c|c|c|c|c|c|c|c|c|}
\hline & INSTITUTION & $\begin{array}{r}\text { LAW LIBRARY } \\
\text { VOLUMES }\end{array}$ & $\begin{array}{r}\text { Total } \\
\text { Volumes }\end{array}$ & $\begin{array}{c}\text { LAW } \% \text { OF } \\
\text { TOTAL }\end{array}$ & & INSTITUTION & $\begin{array}{r}\text { LAW LIBRARY } \\
\text { VOLUMES }\end{array}$ & $\begin{array}{r}\text { Total } \\
\text { Volumes }\end{array}$ & $\begin{array}{c}\text { LAW } \% \text { OF } \\
\text { TOTAL }\end{array}$ \\
\hline 1 & HARVARD & $1,765,543$ & $15,965,675$ & 11 & 39 & COLORADO & 331,485 & $3,843,458$ & 9 \\
\hline 2 & COLUMBIA & 903,410 & $10,004,848$ & 9 & 40 & ALABAMA & 328,668 & $2,835,808$ & 12 \\
\hline 3 & YALE & 895,837 & $12,283,594$ & 7 & 41 & FLORIDA & 322,990 & $4,229,717$ & 8 \\
\hline 4 & IOWA & 798,317 & $4,765,690$ & 17 & 42 & RUTGERS-Camden & 320,218 & $4,462,249$ & 7 \\
\hline 5 & NEW YORK & 781,214 & $4,946,277$ & 16 & 43 & SOUTHERN CALIFORNIA & 313,032 & $4,039,228$ & 8 \\
\hline 6 & TEXAS & 780,177 & $9,323,252$ & 8 & 44 & TULANE & 311,355 & $3,106,645$ & 10 \\
\hline 7 & MINNESOTA & 730,399 & $6,867,777$ & 11 & 45 & FLORIDA STATE & 307,797 & $3,442,420$ & 9 \\
\hline 8 & MICHIGAN & 700,417 & $8,414,070$ & 8 & 46 & CASE WESTERN RESERVE & 306,226 & $2,518,324$ & 12 \\
\hline 9 & CALIFORNIA, BERKELEY & 682,682 & $10,725,334$ & 6 & 47 & SUNY-BUFFALO & 299,979 & $3,655,089$ & 8 \\
\hline 10 & VIRGINIA & 644,112 & $5,465,077$ & 12 & 48 & PENNSYLVANIA STATE & 299,480 & $5,220,080$ & 6 \\
\hline 11 & PENNSYLVANIA & 623,953 & $5,994,465$ & 10 & 49 & CALIFORNIA, DAVIS & 294,938 & $3,599,983$ & 8 \\
\hline 12 & GEORGIA & 623,325 & $4,559,220$ & 14 & 50 & CINCINNATI & 284,250 & $3,418,599$ & 8 \\
\hline 13 & ILLINOIS, URBANA & 622,275 & $10,712,706$ & 6 & 51 & SOUTH CAROLINA & 282,662 & $3,579,504$ & 8 \\
\hline 14 & NORTHWESTERN & 582,332 & $4,768,588$ & 12 & 52 & KANSAS & 277,564 & $4,210,639$ & 7 \\
\hline 15 & OHIO STATE & 576,533 & $6,244,095$ & 9 & 53 & ARIZONA STATE & 272,173 & $4,342,425$ & 6 \\
\hline 16 & CORNELL & 564,386 & $7,999,177$ & 7 & 54 & KENTUCKY & 261,953 & $3,537,710$ & 7 \\
\hline 17 & GEORGETOWN & 562,986 & $2,955,278$ & 19 & 55 & BOSTON COLLEGE & 245,824 & $2,446,744$ & 10 \\
\hline 18 & CHICAGO & 561,065 & $7,926,905$ & 7 & 56 & NEBRASKA & 243,926 & $3,171,134$ & 8 \\
\hline 19 & CALIFORNIA, LOS ANGELES & 556,619 & $8,272,112$ & 7 & 57 & LOUISVILLE & 238,703 & $2,143,282$ & 11 \\
\hline 20 & HOUSTON & 523,350 & $2,558,069$ & 20 & 58 & UTAH & 237,630 & $3,320,167$ & 7 \\
\hline 21 & VANDERBILT & 482,604 & $3,311,665$ & 15 & 59 & NEW MEXICO & 236,734 & $2,707,830$ & 9 \\
\hline 22 & LOUISIANA STATE & 452,841 & $3,548,726$ & 13 & 60 & ARIZONA & 225,943 & $5,632,559$ & 4 \\
\hline 23 & WASHINGTON U.-ST. LOUIS & 451,567 & $4,162,980$ & 11 & 61 & PITTSBURGH & 224,304 & $5,116,305$ & 4 \\
\hline 24 & INDIANA & 449,617 & $7,553,823$ & 6 & 62 & QUEENS & 220,599 & $2,517,328$ & 9 \\
\hline 25 & DUKE & 442,021 & $5,872,136$ & 8 & 63 & SYRACUSE & 218,058 & $3,160,240$ & 7 \\
\hline 26 & MIAMI & 441,835 & $3,227,943$ & 14 & 64 & EMORY & 217,631 & $3,298,971$ & 7 \\
\hline 27 & TEMPLE & 432,753 & $3,137,142$ & 14 & 65 & SOUTHERN ILLINOIS & 215,099 & $3,073,665$ & 7 \\
\hline 28 & WASHINGTON & 429,390 & $7,304,234$ & 6 & 66 & OKLAHOMA & 211,382 & $5,047,646$ & 4 \\
\hline 29 & WISCONSIN & 406,985 & $7,916,419$ & 5 & 67 & HOWARD & 209,500 & $2,467,638$ & 8 \\
\hline 30 & MISSOURI & 389,299 & $3,454,585$ & 11 & 68 & TORONTO & 208,075 & $10,719,219$ & 2 \\
\hline 31 & RUTGERS-Newark & 362,178 & $4,462,249$ & 8 & 69 & TEXAS TECH & 205,121 & $2,578,185$ & 8 \\
\hline 32 & GEORGE WASHINGTON & 356,932 & $2,219,849$ & 16 & 70 & OREGON & 190,206 & $2,905,608$ & 7 \\
\hline 33 & NORTH CAROLINA & 351,410 & $6,154,365$ & 6 & 71 & WESTERN ONTARIO & 184,521 & $3,362,078$ & 5 \\
\hline 34 & CONNECTICUT & 344,598 & $3,637,190$ & 9 & 72 & MONTREAL & 181,968 & $3,150,386$ & 6 \\
\hline 35 & TENNESSEE & 342,537 & $3,251,893$ & 11 & 73 & SASKATCHEWAN & 163,557 & $2,367,250$ & 7 \\
\hline 36 & NOTRE DAME & 342,085 & $3,322,395$ & 10 & 74 & HAWAII & 132,888 & $3,486,937$ & 4 \\
\hline 37 & BRIGHAM YOUNG & 337,197 & $3,908,127$ & 9 & 75 & MICHIGAN STATE & 131,914 & $4,915,621$ & 3 \\
\hline 38 & BOSTON & 332,247 & $2,463,324$ & 13 & & & & & \\
\hline
\end{tabular}


Rank Order Table 2: Volumes Added (Gross)

\begin{tabular}{|c|c|c|c|c|c|c|c|c|c|}
\hline & INSTITUTION & $\begin{array}{r}\text { LAW LIBRARY } \\
\text { Volumes }\end{array}$ & $\begin{array}{r}\text { Total } \\
\text { Volumes }\end{array}$ & $\begin{array}{c}\text { LAW } \% \text { OF } \\
\text { TOTAL }\end{array}$ & & INSTITUTION & $\begin{array}{r}\text { LAW LIBRARY } \\
\text { Volumes }\end{array}$ & $\begin{array}{r}\text { Total } \\
\text { Volumes }\end{array}$ & $\begin{array}{c}\text { LAW } \% \text { OF } \\
\text { TOTAL }\end{array}$ \\
\hline 1 & PENNSYLVANIA STATE & 33,436 & 185,622 & 18 & 39 & QUEENS & 7,823 & 48,389 & 16 \\
\hline 2 & RUTGERS-Newark & 28,941 & 100,692 & 29 & 40 & ALABAMA & 7,793 & 46,035 & 17 \\
\hline 3 & NORTH CAROLINA & 28,938 & 138,562 & 21 & 41 & BRIGHAM YOUNG & 7,258 & 184,608 & 4 \\
\hline 4 & TEMPLE & 28,797 & 66,014 & 44 & 42 & CONNECTICUT & 7,191 & 41,666 & 17 \\
\hline 5 & HARVARD & 25,709 & 347,049 & 7 & 43 & TEXAS TECH & 7,159 & 58,178 & 12 \\
\hline 6 & IOWA & 22,389 & 184,240 & 12 & 44 & ARIZONA & 7,099 & 82,344 & 9 \\
\hline 7 & GEORGETOWN & 21,927 & 91,987 & 24 & 45 & SYRACUSE & 6,307 & 30,294 & 21 \\
\hline 8 & YALE & 19,644 & 254,999 & 8 & 46 & RUTGERS-Camden & 6,299 & 100,692 & 6 \\
\hline 9 & MINNESOTA & 17,140 & 137,273 & 12 & 47 & BOSTON COLLEGE & 6,232 & 50,137 & 12 \\
\hline 10 & MIAMI & 16,868 & 70,498 & 24 & 48 & SOUTHERN ILLINOIS & 6,174 & 29,439 & 21 \\
\hline 11 & MICHIGAN & 14,817 & 157,552 & 9 & 49 & PITTSBURGH & 6,149 & 217,345 & 3 \\
\hline 12 & HOUSTON & 13,722 & 79,824 & 17 & 50 & SOUTHERN CALIFORNIA & 6,146 & 73,605 & 8 \\
\hline 13 & CHICAGO & 13,588 & 191,311 & 7 & 51 & SUNY-BUFFALO & 5,940 & 54,272 & 11 \\
\hline 14 & PENNSYLVANIA & 13,571 & 107,078 & 13 & 52 & CASE WESTERN RESERVE & 5,785 & 27,987 & 21 \\
\hline 15 & INDIANA & 13,433 & 196,277 & 7 & 53 & NEBRASKA & 5,694 & 65,994 & 9 \\
\hline 16 & TEXAS & 13,426 & 114,833 & 12 & 54 & BOSTON & 5,690 & 47,105 & 12 \\
\hline 17 & COLUMBIA & 12,850 & 229,439 & 6 & 55 & CALIFORNIA, DAVIS & 5,650 & 81,543 & 7 \\
\hline 18 & LOUISIANA STATE & 11,772 & 54,400 & 22 & 56 & MICHIGAN STATE & 5,380 & 66,906 & 8 \\
\hline 19 & TENNESSEE & 11,281 & 78,738 & 14 & 57 & UTAH & 5,107 & 69,759 & 7 \\
\hline 20 & WASHINGTON U.-ST. LOUIS & 11,219 & 58,602 & 19 & 58 & FLORIDA & 4,976 & 59,624 & 8 \\
\hline 21 & EMORY & 11,111 & 94,667 & 12 & 59 & ARIZONA STATE & 4,853 & 93,623 & 5 \\
\hline 22 & MISSOURI & 10,966 & 64,014 & 17 & 60 & CINCINNATI & 4,744 & 94,815 & 5 \\
\hline 23 & NORTHWESTERN & 10,785 & 92,004 & 12 & 61 & SASKATCHEWAN & 4,195 & 63,731 & 7 \\
\hline 24 & WISCONSIN & 10,717 & 116,200 & 9 & 62 & OKLAHOMA & 3,912 & 131,123 & 3 \\
\hline 25 & GEORGIA & 10,678 & 75,494 & 14 & 63 & KENTUCKY & 3,862 & 85,561 & 5 \\
\hline 26 & ILLINOIS, URBANA & 10,643 & 196,882 & 5 & 64 & FLORIDA STATE & 3,680 & 62,369 & 6 \\
\hline 27 & VIRGINIA & 10,640 & 88,756 & 12 & 65 & KANSAS & 3,573 & 62,438 & 6 \\
\hline 28 & CALIFORNIA, LOS ANGELES & 10,454 & 131,198 & 8 & 66 & MONTREAL & 3,554 & 74,411 & 5 \\
\hline 29 & DUKE & 10,152 & 98,905 & 10 & 67 & HAWAII & 3,263 & 56,308 & 6 \\
\hline 30 & OHIO STATE & 9,779 & 115,400 & 8 & 68 & SOUTH CAROLINA & 3,192 & 54,301 & 6 \\
\hline 31 & COLORADO & 9,700 & 99,592 & 10 & 69 & TORONTO & 3,114 & 232,020 & 1 \\
\hline 32 & CALIFORNIA, BERKELEY & 9,677 & 202,625 & 5 & 70 & OREGON & 2,968 & 62,013 & 5 \\
\hline 33 & NOTRE DAME & 9,657 & 79,540 & 12 & 71 & TULANE & 2,696 & 44,108 & 6 \\
\hline 34 & VANDERBILT & 9,379 & 58,102 & 16 & 72 & HOWARD & 2,378 & 33,864 & 7 \\
\hline 35 & GEORGE WASHINGTON & 8,857 & 46,166 & 19 & 73 & LOUISVILLE & 2,015 & 63,740 & 3 \\
\hline 36 & CORNELL & 8,670 & 128,113 & 7 & 74 & NEW MEXICO & 1,189 & 62,075 & 2 \\
\hline 37 & WASHINGTON & 8,669 & 221,816 & 4 & 75 & WESTERN ONTARIO & 921 & 52,753 & 2 \\
\hline 38 & NEW YORK & 8,399 & 100,043 & 8 & & & & & \\
\hline
\end{tabular}


Rank Order Table 3: Current Serials (Total)

\begin{tabular}{|c|c|c|c|c|c|c|c|c|c|}
\hline & INSTITUTION & $\begin{array}{r}\text { LaW Library } \\
\text { SERIaLS }\end{array}$ & $\begin{array}{r}\text { Total } \\
\text { Serials }\end{array}$ & $\begin{array}{r}\text { LAW } \% \text { OF } \\
\text { TOTAL }\end{array}$ & & INSTITUTION & $\begin{array}{r}\text { LaW Library } \\
\text { Serials }\end{array}$ & $\begin{array}{r}\text { TOtal } \\
\text { SERIaLS }\end{array}$ & $\begin{array}{l}\text { LAW } \% \text { OF } \\
\text { TOTAL }\end{array}$ \\
\hline 1 & PENNSYLVANIA & 41,411 & 84,182 & 49 & 39 & NEW YORK & 5,046 & 58,968 & 9 \\
\hline 2 & NOTRE DAME & 37,067 & 68,038 & 54 & 40 & RUTGERS-Newark & 4,911 & 54,450 & 9 \\
\hline 3 & GEORGIA & 32,818 & 80,976 & 41 & 41 & UTAH & 4,860 & 45,830 & 11 \\
\hline 4 & CORNELL & 25,000 & 92,000 & 27 & 42 & FLORIDA STATE & 4,800 & 51,733 & 9 \\
\hline 5 & GEORGE WASHINGTON & 21,102 & 65,604 & 32 & 43 & BOSTON & 4,748 & 45,264 & 10 \\
\hline 6 & HARVARD & 15,725 & 110,463 & 14 & 44 & TEXAS TECH & 4,713 & 70,114 & 7 \\
\hline 7 & MICHIGAN STATE & 12,494 & 74,177 & 17 & 45 & COLORADO & 4,513 & 50,350 & 9 \\
\hline 8 & INDIANA & 11,927 & 57,534 & 21 & 46 & HOWARD & 4,321 & 16,627 & 26 \\
\hline 9 & IOWA & 10,101 & 59,380 & 17 & 47 & BRIGHAM YOUNG & 4,264 & 54,183 & 8 \\
\hline 10 & YALE & 9,858 & 89,649 & 11 & 48 & WASHINGTON U.-ST. LOUIS & 4,229 & 49,970 & 8 \\
\hline 11 & EMORY & 9,841 & 54,218 & 18 & 49 & PITTSBURGH & 4,103 & 48,637 & 8 \\
\hline 12 & GEORGETOWN & 8,672 & 53,697 & 16 & 50 & WASHINGTON & 4,089 & 61,272 & 7 \\
\hline 13 & OHIO STATE & 8,453 & 68,550 & 12 & 51 & ILLINOIS, URBANA & 4,055 & 107,666 & 4 \\
\hline 14 & CHICAGO & 8,380 & 57,236 & 15 & 52 & BOSTON COLLEGE & 3,894 & 31,714 & 12 \\
\hline 15 & CALIFORNIA, LOS ANGELES & 8,283 & 76,248 & 11 & 53 & KANSAS & 3,526 & 48,212 & 7 \\
\hline 16 & SOUTHERN CALIFORNIA & 8,071 & 89,971 & 9 & 54 & TEMPLE & 3,451 & 51,198 & 7 \\
\hline 17 & FLORIDA & 8,067 & 73,747 & 11 & 55 & ALABAMA & 3,415 & 44,079 & 8 \\
\hline 18 & MIAMI & 8,032 & 62,621 & 13 & 56 & DUKE & 3,411 & 47,122 & 7 \\
\hline 19 & PENNSYLVANIA STATE & 7,216 & 68,876 & 10 & 57 & NEW MEXICO & 3,296 & 40,374 & 8 \\
\hline 20 & TEXAS & 7,191 & 56,097 & 13 & 58 & SOUTH CAROLINA & 3,126 & 51,540 & 6 \\
\hline 21 & NORTH CAROLINA & 6,932 & 56,647 & 12 & 59 & ARIZONA & 2,891 & 75,181 & 4 \\
\hline 22 & VANDERBILT & 6,867 & 44,199 & 16 & 60 & MONTREAL & 2,793 & 54,265 & 5 \\
\hline 23 & LOUISIANA STATE & 6,835 & 86,026 & 8 & 61 & NEBRASKA & 2,724 & 41,034 & 7 \\
\hline 24 & COLUMBIA & 6,773 & 119,637 & 6 & 62 & HOUSTON & 2,221 & 42,812 & 5 \\
\hline 25 & CINCINNATI & 6,627 & 69,609 & 10 & 63 & SYRACUSE & 2,190 & 23,285 & 9 \\
\hline 26 & CALIFORNIA, BERKELEY & 6,626 & 87,265 & 8 & 64 & TENNESSEE & 2,114 & 44,337 & 5 \\
\hline 27 & MICHIGAN & 6,263 & 71,788 & 9 & 65 & SASKATCHEWAN & 2,056 & 34,920 & 6 \\
\hline 28 & HAWAII & 6,262 & 58,111 & 11 & 66 & TORONTO & 1,902 & 47,916 & 4 \\
\hline 29 & CONNECTICUT & 6,173 & 51,007 & 12 & 67 & OREGON & 1,732 & 62,348 & 3 \\
\hline 30 & WISCONSIN & 6,074 & 54,164 & 11 & 68 & SOUTHERN ILLINOIS & 1,533 & 39,221 & 4 \\
\hline 31 & CASE WESTERN RESERVE & 5,957 & 54,252 & 11 & 69 & WESTERN ONTARIO & 1,400 & 56,223 & 2 \\
\hline 32 & SUNY-BUFFALO & 5,659 & 71,234 & 8 & 70 & MISSOURI & 213 & 52,490 & 0 \\
\hline 33 & KENTUCKY & 5,650 & 44,610 & 13 & 71 & ARIZONA STATE & $\mathrm{UA} / \mathrm{NA}$ & 82,862 & $\mathrm{UA} / \mathrm{NA}$ \\
\hline 34 & LOUISVILLE & 5,420 & 39,268 & 14 & 72 & MINNESOTA & $\mathrm{UA} / \mathrm{NA}$ & 77,446 & $\mathrm{UA} / \mathrm{NA}$ \\
\hline 35 & NORTHWESTERN & 5,337 & 64,888 & 8 & 73 & OKLAHOMA & $\mathrm{UA} / \mathrm{NA}$ & 55,815 & $\mathrm{UA} / \mathrm{NA}$ \\
\hline 36 & TULANE & 5,277 & 34,820 & 15 & 74 & QUEENS & $\mathrm{UA} / \mathrm{NA}$ & 39,823 & $\mathrm{UA} / \mathrm{NA}$ \\
\hline 37 & CALIFORNIA, DAVIS & 5,227 & 42,592 & 12 & 75 & VIRGINIA & $\mathrm{UA} / \mathrm{NA}$ & 82,440 & $\mathrm{UA} / \mathrm{NA}$ \\
\hline 38 & RUTGERS-Camden & 5,116 & 54,450 & 9 & & & & & \\
\hline
\end{tabular}


Rank Order Table 4: Total Library Expenditures

\begin{tabular}{|c|c|c|c|c|c|c|c|c|c|}
\hline & INSTITUTION & $\begin{array}{r}\text { LAW LIBRARY } \\
\text { EXPENDITURES }\end{array}$ & $\begin{array}{c}\text { TOTAL LIBRARY } \\
\text { EXPENDITURES }\end{array}$ & $\begin{array}{r}\text { LAW } \% \text { OF } \\
\text { TOTAL }\end{array}$ & & INSTITUTION & $\begin{array}{r}\text { LAW LIBRARY } \\
\text { EXPENDITURES }\end{array}$ & $\begin{array}{r}\text { TOTAL LIBRARY } \\
\text { EXPENDITURES } \\
\end{array}$ & $\begin{array}{r}\text { LAW \% OF } \\
\text { TOTAL } \\
\end{array}$ \\
\hline 1 & HARVARD & $10,651,639$ & $110,849,458$ & 10 & 39 & LOUISIANA STATE & $2,463,977$ & $14,526,765$ & 17 \\
\hline 2 & GEORGETOWN & $7,294,270$ & $24,299,359$ & 30 & 40 & VANDERBILT & $2,424,916$ & $24,673,090$ & 10 \\
\hline 3 & CALIFORNIA, BERKELEY & $6,156,081$ & $53,231,754$ & 12 & 41 & TENNESSEE & $2,419,620$ & $22,810,227$ & 11 \\
\hline 4 & YALE & $6,116,248$ & $77,403,912$ & 8 & 42 & ALABAMA & $2,415,430$ & $15,501,146$ & 16 \\
\hline 5 & GEORGE WASHINGTON & $5,467,718$ & $23,153,326$ & 24 & 43 & LOUISVILLE & $2,291,201$ & $16,560,259$ & 14 \\
\hline 6 & MICHIGAN & $5,332,788$ & $50,591,407$ & 11 & 44 & SYRACUSE & $2,291,101$ & $16,849,931$ & 14 \\
\hline 7 & NEW YORK & $5,014,363$ & $42,669,230$ & 12 & 45 & ARIZONA & $2,129,926$ & $30,769,327$ & 7 \\
\hline 8 & COLUMBIA & $4,962,719$ & $60,071,960$ & 8 & 46 & HOUSTON & $2,128,130$ & $17,117,492$ & 12 \\
\hline 9 & IOWA & $4,623,125$ & $26,647,407$ & 17 & 47 & CALIFORNIA, DAVIS & $2,095,408$ & $21,002,377$ & 10 \\
\hline 10 & MINNESOTA & $3,970,397$ & $39,927,096$ & 10 & 48 & RUTGERS-Newark & $1,986,325$ & $30,653,693$ & 6 \\
\hline 11 & CALIFORNIA, LOS ANGELES & $3,948,993$ & $51,792,128$ & 8 & 49 & SOUTH CAROLINA & $1,947,888$ & $20,943,404$ & 9 \\
\hline 12 & MIAMI & $3,710,964$ & $25,941,411$ & 14 & 50 & ILLINOIS, URBANA & $1,934,500$ & $41,919,073$ & 5 \\
\hline 13 & PENNSYLVANIA & $3,672,392$ & $35,258,073$ & 10 & 51 & NEW MEXICO & $1,931,114$ & $22,653,088$ & 9 \\
\hline 14 & CHICAGO & $3,504,971$ & $31,966,296$ & 11 & 52 & BRIGHAM YOUNG & $1,922,511$ & $25,159,442$ & 8 \\
\hline 15 & TEXAS & $3,501,514$ & $45,044,095$ & 8 & 53 & ARIZONA STATE & $1,919,308$ & $27,730,343$ & 7 \\
\hline 16 & NOTRE DAME & $3,237,349$ & $23,257,537$ & 14 & 54 & TORONTO & $1,903,034$ & $60,344,407$ & 3 \\
\hline 17 & WASHINGTON & $3,125,278$ & $41,583,736$ & 8 & 55 & MICHIGAN STATE & $1,882,509$ & $24,458,354$ & 8 \\
\hline 18 & DUKE & $3,117,265$ & $35,769,252$ & 9 & 56 & PITTSBURGH & $1,865,144$ & $29,890,593$ & 6 \\
\hline 19 & VIRGINIA & $3,078,716$ & $34,444,481$ & 9 & 57 & UTAH & $1,855,171$ & $24,641,932$ & 8 \\
\hline 20 & CONNECTICUT & $3,031,202$ & $25,071,952$ & 12 & 58 & PENNSYLVANIA STATE & $1,850,103$ & $50,251,356$ & 4 \\
\hline 21 & NORTHWESTERN & $2,969,717$ & $27,272,613$ & 11 & 59 & RUTGERS-Camden & $1,812,459$ & $30,653,693$ & 6 \\
\hline 22 & BOSTON COLLEGE & $2,923,736$ & $18,217,616$ & 16 & 60 & FLORIDA STATE & $1,798,231$ & $15,197,764$ & 12 \\
\hline 23 & BOSTON & $2,895,157$ & $18,916,916$ & 15 & 61 & CINCINNATI & $1,785,903$ & $22,045,924$ & 8 \\
\hline 24 & TEMPLE & $2,839,837$ & $21,280,634$ & 13 & 62 & TULANE & $1,711,272$ & $13,974,872$ & 12 \\
\hline 25 & WASHINGTON U.-ST. LOUIS & $2,825,583$ & $27,424,013$ & 10 & 63 & KENTUCKY & $1,648,228$ & $20,920,715$ & 8 \\
\hline 26 & CASE WESTERN RESERVE & $2,815,576$ & $13,382,448$ & 21 & 64 & OREGON & $1,622,509$ & $14,988,631$ & 11 \\
\hline 27 & NORTH CAROLINA & $2,805,058$ & $35,425,976$ & 8 & 65 & HAWAII & $1,617,779$ & $18,534,243$ & 9 \\
\hline 28 & FLORIDA & $2,783,470$ & $27,443,254$ & 10 & 66 & OKLAHOMA & $1,553,934$ & $20,844,144$ & 7 \\
\hline 29 & CORNELL & $2,719,470$ & $43,832,341$ & 6 & 67 & SOUTHERN ILLINOIS & $1,541,263$ & $14,564,091$ & 11 \\
\hline 30 & TEXAS TECH & $2,684,314$ & $22,635,446$ & 12 & 68 & HOWARD & $1,432,847$ & $9,916,198$ & 14 \\
\hline 31 & OHIO STATE & $2,638,329$ & $32,480,575$ & 8 & 69 & MONTREAL & $1,395,665$ & $28,153,803$ & 5 \\
\hline 32 & INDIANA & $2,631,425$ & $33,641,171$ & 8 & 70 & MISSOURI & $1,270,868$ & $16,698,370$ & 8 \\
\hline 33 & SUNY-BUFFALO & $2,581,823$ & $20,054,901$ & 13 & 71 & KANSAS & $1,262,099$ & $21,156,733$ & 6 \\
\hline 34 & GEORGIA & $2,571,773$ & $23,703,488$ & 11 & 72 & NEBRASKA & $1,171,496$ & $14,632,237$ & 8 \\
\hline 35 & WISCONSIN & $2,550,549$ & $41,536,552$ & 6 & 73 & SASKATCHEWAN & $1,134,558$ & $22,127,910$ & 5 \\
\hline 36 & SOUTHERN CALIFORNIA & $2,539,166$ & $34,881,430$ & 7 & 74 & QUEENS & $1,069,042$ & $16,884,844$ & 6 \\
\hline 37 & EMORY & $2,530,843$ & $33,741,980$ & 8 & 75 & WESTERN ONTARIO & $1,044,537$ & $18,773,453$ & 6 \\
\hline 38 & COLORADO & $2,466,438$ & $19,555,663$ & 13 & & & & & \\
\hline
\end{tabular}


Rank Order Table 5: Total StafF

\begin{tabular}{|c|c|c|c|c|c|c|c|c|c|}
\hline & INSTITUTION & $\begin{array}{r}\text { LAW Library } \\
\text { StafF }\end{array}$ & $\begin{array}{l}\text { Total } \\
\text { StafF }\end{array}$ & $\begin{array}{c}\text { LAW } \% \text { OF } \\
\text { TOTAL }\end{array}$ & & INSTITUTION & $\begin{array}{r}\text { LAW LiBRARY } \\
\text { StafF }\end{array}$ & $\begin{array}{l}\text { TOTAL } \\
\text { StafF }\end{array}$ & $\begin{array}{l}\text { LAW } \% \text { OF } \\
\text { TOTAL }\end{array}$ \\
\hline 1 & HARVARD & 123 & 1,277 & 10 & 39 & WASHINGTON U.-ST. LOUIS & 26 & 269 & 10 \\
\hline 2 & GEORGETOWN & 86 & 247 & 35 & 40 & TEMPLE & 25 & 227 & 11 \\
\hline 3 & NEW YORK & 63 & 443 & 14 & 41 & ARIZONA STATE & 24 & 338 & 7 \\
\hline 4 & YALE & 59 & 705 & 8 & 42 & INDIANA & 24 & 483 & 5 \\
\hline 5 & CALIFORNIA, BERKELEY & 57 & 584 & 10 & 43 & GEORGIA & 23 & 303 & 8 \\
\hline 6 & MICHIGAN & 53 & 585 & 9 & 44 & SOUTHERN CALIFORNIA & 23 & 386 & 6 \\
\hline 7 & COLUMBIA & 49 & 716 & 7 & 45 & FLORIDA STATE & 22 & 289 & 8 \\
\hline 8 & GEORGE WASHINGTON & 47 & 279 & 17 & 46 & ILLINOIS, URBANA & 22 & 533 & 4 \\
\hline 9 & FLORIDA & 40 & 426 & 9 & 47 & OHIO STATE & 22 & 423 & 5 \\
\hline 10 & TEXAS & 40 & 551 & 7 & 48 & OKLAHOMA & 22 & 233 & 9 \\
\hline 11 & IOWA & 39 & 283 & 14 & 49 & UTAH & 22 & 358 & 6 \\
\hline 12 & LOUISIANA STATE & 36 & 192 & 19 & 50 & ALABAMA & 21 & 207 & 10 \\
\hline 13 & MINNESOTA & 36 & 402 & 9 & 51 & EMORY & 21 & 307 & 7 \\
\hline 14 & PENNSYLVANIA & 36 & 414 & 9 & 52 & KANSAS & 21 & 281 & 7 \\
\hline 15 & BRIGHAM YOUNG & 34 & 389 & 9 & 53 & MISSOURI & 21 & 214 & 10 \\
\hline 16 & CALIFORNIA, LOS ANGELES & 34 & 606 & 6 & 54 & RUTGERS-Camden & 21 & 415 & 5 \\
\hline 17 & MIAMI & 34 & 259 & 13 & 55 & RUTGERS-Newark & 21 & 415 & 5 \\
\hline 18 & TENNESSEE & 33 & 273 & 12 & 56 & TULANE & 21 & 150 & 14 \\
\hline 19 & WASHINGTON & 33 & 513 & 6 & 57 & COLORADO & 20 & 208 & 10 \\
\hline 20 & NOTRE DAME & 32 & 263 & 12 & 58 & MICHIGAN STATE & 20 & 296 & 7 \\
\hline 21 & TEXAS TECH & 32 & 309 & 10 & 59 & NEW MEXICO & 20 & 266 & 8 \\
\hline 22 & VIRGINIA & 32 & 371 & 9 & 60 & PITTSBURGH & 20 & 363 & 6 \\
\hline 23 & BOSTON & 31 & 289 & 11 & 61 & SOUTHERN ILLINOIS & 20 & 202 & 10 \\
\hline 24 & NORTHWESTERN & 31 & 336 & 9 & 62 & VANDERBILT & 20 & 248 & 8 \\
\hline 25 & WISCONSIN & 31 & 643 & 5 & 63 & CALIFORNIA, DAVIS & 19 & 273 & 7 \\
\hline 26 & CHICAGO & 30 & 320 & 9 & 64 & OREGON & 18 & 213 & 8 \\
\hline 27 & HOWARD & 30 & 166 & 18 & 65 & CINCINNATI & 17 & 205 & 8 \\
\hline 28 & SUNY-BUFFALO & 30 & 257 & 12 & 66 & NEBRASKA & 17 & 202 & 8 \\
\hline 29 & BOSTON COLLEGE & 29 & 200 & 15 & 67 & SOUTH CAROLINA & 17 & 278 & 6 \\
\hline 30 & HOUSTON & 29 & 200 & 15 & 68 & TORONTO & 17 & 670 & 3 \\
\hline 31 & CASE WESTERN RESERVE & 28 & 137 & 20 & 69 & KENTUCKY & 16 & 277 & 6 \\
\hline 32 & PENNSYLVANIA STATE & 28 & 622 & 5 & 70 & LOUISVILLE & 16 & 190 & 8 \\
\hline 33 & ARIZONA & 26 & 332 & 8 & 71 & MONTREAL & 16 & 391 & 4 \\
\hline 34 & CONNECTICUT & 26 & 212 & 12 & 72 & HAWAII & 15 & 255 & 6 \\
\hline 35 & CORNELL & 26 & 548 & 5 & 73 & QUEENS & 12 & 165 & 7 \\
\hline 36 & DUKE & 26 & 371 & 7 & 74 & SASKATCHEWAN & 9 & 177 & 5 \\
\hline 37 & NORTH CAROLINA & 26 & 446 & 6 & 75 & WESTERN ONTARIO & 9 & 194 & 5 \\
\hline 38 & SYRACUSE & 26 & 232 & 11 & & & & & \\
\hline
\end{tabular}


Rank Order Table 6: Total Electronic Resources

\begin{tabular}{|c|c|c|c|c|c|c|c|c|c|}
\hline & INSTITUTION & $\begin{array}{l}\text { LAW LIBRARY } \\
\text { E-RESOURCES }\end{array}$ & $\begin{array}{r}\text { Total } \\
\text { E-Resources }\end{array}$ & $\begin{array}{c}\text { LAW } \% \text { OF } \\
\text { TOTAL }\end{array}$ & & INSTITUTION & $\begin{array}{l}\text { LAW LIBRARY } \\
\text { E-RESOURCES }\end{array}$ & $\begin{array}{r}\text { Total } \\
\text { E-Resources }\end{array}$ & $\begin{array}{c}\text { LAW } \% \text { OF } \\
\text { TOTAL }\end{array}$ \\
\hline 1 & PENNSYLVANIA STATE & 599,467 & $9,448,072$ & 6 & 39 & SYRACUSE & 224,080 & $3,350,462$ & 7 \\
\hline 2 & NEW YORK & 574,361 & $7,903,569$ & 7 & 40 & WISCONSIN & 208,515 & $5,094,585$ & 4 \\
\hline 3 & VANDERBILT & 560,822 & $6,954,968$ & 8 & 41 & CASE WESTERN RESERVE & 206,705 & $3,209,445$ & 6 \\
\hline 4 & GEORGIA & 556,947 & $5,947,356$ & 9 & 42 & ALABAMA & 206,181 & $3,487,313$ & 6 \\
\hline 5 & FLORIDA & 513,398 & $4,940,171$ & 10 & 43 & DUKE & 204,091 & $6,758,268$ & 3 \\
\hline 6 & GEORGETOWN & 489,676 & $3,979,582$ & 12 & 44 & TENNESSEE & 199,229 & $5,173,801$ & 4 \\
\hline 7 & WASHINGTON U.-ST. LOUIS & 447,698 & $5,019,863$ & 9 & 45 & NEBRASKA & 194,375 & $2,200,652$ & 9 \\
\hline 8 & GEORGE WASHINGTON & 427,412 & $3,660,783$ & 12 & 46 & YALE & 190,447 & $6,991,200$ & 3 \\
\hline 9 & NOTRE DAME & 415,374 & $5,238,495$ & 8 & 47 & PENNSYLVANIA & 188,498 & $5,558,118$ & 3 \\
\hline 10 & HARVARD & 372,874 & $6,681,995$ & 6 & 48 & OREGON & 185,893 & $2,418,763$ & 8 \\
\hline 11 & TEMPLE & 365,879 & $6,542,673$ & 6 & 49 & WASHINGTON & 185,530 & $6,818,776$ & 3 \\
\hline 12 & COLUMBIA & 359,720 & $9,724,550$ & 4 & 50 & CONNECTICUT & 181,468 & $5,172,502$ & 4 \\
\hline 13 & MIAMI & 357,162 & $7,323,132$ & 5 & 51 & VIRGINIA & 180,773 & $4,476,702$ & 4 \\
\hline 14 & TULANE & 350,574 & $3,144,786$ & 11 & 52 & CALIFORNIA, DAVIS & 179,313 & $3,822,482$ & 5 \\
\hline 15 & MICHIGAN STATE & 326,418 & $5,438,142$ & 6 & 53 & RUTGERS-Newark & 175,244 & $3,721,706$ & 5 \\
\hline 16 & TEXAS TECH & 325,522 & $3,131,537$ & 10 & 54 & ILLINOIS, URBANA & 147,573 & $5,369,858$ & 3 \\
\hline 17 & BRIGHAM YOUNG & 317,068 & $2,678,255$ & 12 & 55 & HAWAII & 134,999 & $3,926,834$ & 3 \\
\hline 18 & INDIANA & 304,212 & $5,780,889$ & 5 & 56 & MONTREAL & 118,592 & $5,228,750$ & 2 \\
\hline 19 & TEXAS & 298,384 & $8,976,961$ & 3 & 57 & SOUTHERN ILLINOIS & 115,959 & $3,317,029$ & 3 \\
\hline 20 & ARIZONA STATE & 288,328 & $6,268,359$ & 5 & 58 & NEW MEXICO & 105,833 & $3,928,276$ & 3 \\
\hline 21 & PITTSBURGH & 285,212 & $8,829,087$ & 3 & 59 & RUTGERS-Camden & 103,044 & $3,721,706$ & 3 \\
\hline 22 & OKLAHOMA & 283,593 & $3,836,788$ & 7 & 60 & KENTUCKY & 100,880 & $3,779,661$ & 3 \\
\hline 23 & FLORIDA STATE & 279,356 & $4,627,530$ & 6 & 61 & CINCINNATI & 94,838 & $5,178,257$ & 2 \\
\hline 24 & MINNESOTA & 276,631 & $6,445,354$ & 4 & 62 & OHIO STATE & 93,617 & $5,111,173$ & 2 \\
\hline 25 & SUNY-BUFFALO & 270,263 & $5,113,830$ & 5 & 63 & HOWARD & 92,118 & $1,766,872$ & 5 \\
\hline 26 & NORTHWESTERN & 266,908 & $6,424,824$ & 4 & 64 & CORNELL & 91,323 & $6,080,535$ & 2 \\
\hline 27 & QUEENS & 247,464 & $37,643,961$ & 1 & 65 & LOUISVILLE & 90,514 & $2,678,145$ & 3 \\
\hline 28 & COLORADO & 245,524 & $5,611,532$ & 4 & 66 & BOSTON COLLEGE & 85,533 & $3,271,201$ & 3 \\
\hline 29 & MICHIGAN & 245,150 & $5,854,769$ & 4 & 67 & UTAH & 83,929 & $1,337,771$ & 6 \\
\hline 30 & NORTH CAROLINA & 244,623 & $3,624,155$ & 7 & 68 & TORONTO & 73,935 & $3,460,817$ & 2 \\
\hline 31 & IOWA & 241,454 & $5,590,482$ & 4 & 69 & SOUTHERN CALIFORNIA & 56,325 & $6,739,328$ & 1 \\
\hline 32 & HOUSTON & 240,447 & $2,675,759$ & 9 & 70 & ARIZONA & UA/NA & $6,592,104$ & $\mathrm{UA} / \mathrm{NA}$ \\
\hline 33 & EMORY & 235,332 & $3,995,020$ & 6 & 70 & CALIFORNIA, BERKELEY & 0 & $5,255,796$ & 0 \\
\hline 34 & MISSOURI & 234,785 & $5,388,693$ & 4 & 70 & CALIFORNIA, LOS ANGELES & $\mathrm{UA} / \mathrm{NA}$ & $4,352,521$ & $\mathrm{UA} / \mathrm{NA}$ \\
\hline 35 & SOUTH CAROLINA & 230,856 & $4,078,816$ & 6 & 70 & KANSAS & $\mathrm{UA} / \mathrm{NA}$ & $2,802,454$ & $\mathrm{UA} / \mathrm{NA}$ \\
\hline 36 & LOUISIANA STATE & 229,723 & $3,510,904$ & 7 & 70 & SASKATCHEWAN & $\mathrm{UA} / \mathrm{NA}$ & $6,369,649$ & $\mathrm{UA} / \mathrm{NA}$ \\
\hline 37 & BOSTON & 226,655 & $2,271,508$ & 10 & 70 & WESTERN ONTARIO & $\mathrm{UA} / \mathrm{NA}$ & $6,186,010$ & $\mathrm{UA} / \mathrm{NA}$ \\
\hline 38 & CHICAGO & 224,438 & $5,412,597$ & 4 & & & & & \\
\hline
\end{tabular}




\section{ARL Statistics Questionnaire, 2006-2007 INSTRUCTIONS FOR COMPLETING THE QUESTIONNAIRE}

\section{General Instructions}

Definitions of statistical categories can be found in NISO Z39.7-2004, Information Services and Use: Metrics \& Statistics for Libraries and Information Providers--Data Dictionary (http://www.niso.org/emetrics/current/index.html). ARL has been modifying the interpretation of the standard definitions to address questions posed by by the library staff at various member institutions that complete the survey and with feedback from the ARL Statistics and Assessment Committee (http:// www.arl.org/stats/program/meeting.html).

Please do not use decimals. All figures should be rounded to the nearest whole number.

Please respond to every question. If an exact figure cannot be provided, use NA/UA to indicate that the figure is either unavailable or not applicable. If the appropriate answer is zero or none, use $\mathbf{0 .}$

Although the form allows for data to be entered from both main and branch campuses, an effort should be made to report figures for the main campus only. (The U.S. National Center for Education Statistics, Integrated Postsecondary Education Data System (IPEDS) defines a branch institution as "a campus or site of an educational institution that is not temporary, is located in a community beyond a reasonable commuting distance from its parent institution, and offers organized programs of study, not just courses.") If figures for libraries located at branch campuses are reported, please specify which branch libraries are included and which ones are excluded in the notes below.

A branch library is defined as an auxiliary library service outlet with quarters separate from the central library of an institution, which has a basic collection of books and other materials, a regular staffing level, and an established schedule. A branch library is administered either by the central library or (as in the case of some law and medical libraries) through the administrative structure of other units within the university. Departmental study/reading rooms are not included.

The questionnaire assumes a fiscal year ending June 30, 2007. If your fiscal year is different, please indicate this in the notes below by adjusting the reporting period.

Footnotes. Explanatory footnotes will be included with the published statistics. Provide any notes you may have in the footnotes area at the end of the survey. Reporting libraries are urged to record there any information that would clarify the figures submitted in that line, e.g., the inclusion and exclusion of branch campus libraries. Please make an effort to word your footnotes in a manner consistent with notes appearing in the published report, so that the ARL Office can interpret your footnotes correctly.

\section{Specific Instructions}

Question 1. Volumes in Library. Use the ANSI/ NISO Z39.7-2004 definition for volume as follows:

a single physical unit of any printed, typewritten, handwritten, mimeographed, or processed work, distinguished from other units by a separate binding, encasement, portfolio, or other clear distinction, which has been cataloged, classified, and made ready for use, and which is typically the unit used to charge circulation transactions. Either a serial volume is bound, or it comprises the serial issues that would be bound together if the library bound all serials. 
Include duplicates and bound volumes of periodicals. For purposes of this questionnaire, unclassified bound serials arranged in alphabetical order are considered classified. Exclude microforms, maps, nonprint materials, and uncataloged items. If any of these items cannot be excluded, please provide an explanatory footnote

Include government document volumes that are accessible through the library's catalogs regardless of whether they are separately shelved. "Classified" includes documents arranged by Superintendent of Documents, CODOC, or similar numbers. "Cataloged" includes documents for which records are provided by the library or downloaded from other sources into the library's card or online catalogs. Documents should, to the extent possible, be counted as they would if they were in bound volumes (e.g., 12 issues of an annual serial would be one or two volumes). Title and piece counts should not be considered the same as volume counts. If a volume count has not been kept, it may be estimated through sampling a representative group of title records and determining the corresponding number of volumes, then extrapolating to the rest of the collection. As an alternative, an estimate may be made using the following formulae:

\section{2 documents pieces per foot \\ 10 "traditional" volumes per foot \\ 5.2 documents pieces per volume}

Include e-book units, as long as these e-books are owned or leased and have been cataloged by your library. Include electronic books purchased through vendors such as NetLibrary® or Books $24 \times 7$, and e-books that come as part of aggregate services. Include individual titles of e-book sets that are treated as individual reference sources. Include locally digitized electronic books and electronic theses and dissertations. Provide a footnote explaining how many e-books you are reporting, preferably by specifying the products and the number of titles in a note.

Include volumes purchased collectively where the cost is shared at the time of purchase.

If either formulas or sampling are used for deriving your count, please indicate in a footnote.

Question 1b. Volumes Added. Include only volumes cataloged, classified, and made ready for use. Include government documents if they have been included in the count of volumes on line 1a. Do not include as part of Volumes Added Gross any government documents or other collections (such as large gift collections or e-book packages) that were added to the collection as the result of a one time download or addition to the OPAC. Include these items in Volumes Held of the previous year (Line 1a) and provide a footnote explaining the revision of Line 1a.

Question 2. Monographic Volumes Purchased. Report number of volumes purchased; do not include volumes received or cataloged. Include all volumes for which an expenditure was made during 2006-07, including volumes paid for in advance but not received during the fiscal year. Include monographs in series and continuations. Include e-books that fit the NetLibrary ${ }^{\circledR}$ model, i.e., electronic manifestations of physical entities and/ or units; provide a footnote explaining how many e-books you are reporting, preferably by specifying the products and the number of titles. If only number of titles purchased can be reported, please report the data and provide an explanatory footnote.

Question 3: Basis of Volume Count. A physical count is a piece count; a bibliographic count is a catalog record count.

Questions 4-5. Serials. Use the following definition for a serial:

a publication in any medium issued in successive parts bearing numerical or chronological designations and intended to be continued indefinitely. This definition includes periodicals, newspapers, and annuals (reports, yearbooks, etc.); the journals, memoirs, proceedings, transactions, etc. of societies; and numbered monographic series. 
Report the total number of unique serial titles, NOT SUBSCRIPTIONS, that you currently acquire. Do not include duplicate counts of serial titles. To the extent possible, report all government document serials even if housed in a separate documents collection. Verify the inclusion or exclusion of document serials in Question 5. Exclude unnumbered monographic and publishers' series. Electronic serials acquired as part of a bundle or an aggregated package should be counted by title, even if they are not cataloged, as long as they are made accessible by the library.

Question 4a. Serial titles currently purchased. In the case of consortial agreements, count under 'serial titles currently purchased' those titles for which the library pays any amount from its budgeted expenditures. Include all titles that are part of bundles or aggregated packages, even if your library makes a partial payment for access to those titles. If a purchased title includes electronic access to the title, count that title ONLY ONCE (DEDUPED) as electronic only. If a database includes full-text and abstracted titles, the number of full-text titles can be counted.

Question 4b. Serial titles: Not Purchased. If serial titles have been purchased through a consortium whose budget is centrally funded and independent from the library's budget, these serials should be reported under 'serial titles currently received but not purchased.'

Report other titles that your library receives and does not pay for directly under 'serial titles received but not purchased.

Record those serial titles received without purchase for whatever reason. If within a purchased or aggregated package it cannot be determined that some titles are not purchased, report all titles as purchased.

If separate counts of nonpurchased and purchased serial titles are not available, report only the total number of serial titles currently purchased and received on line 4, and report U/A for lines $4 \mathrm{a}$ and $4 \mathrm{~b}$.

Question 6. Microforms. Report the total number of physical units: reels of microfilm, microcards, and microprint and microfiche sheets. Include all government documents in microform; provide a footnote if documents are excluded.

Question 7. Government documents. Report the total number of physical units (pieces) of government documents in paper format that have not been counted elsewhere. Include local, state, national, and international documents; include documents purchased from a commercial source if shelved with separate documents collections and not counted above. Include serials and monographs. To estimate pieces from a measurement of linear feet, use the formula 1 foot $=52$ pieces and indicate in a footnote that the count is based on this estimate. Exclude microforms and nonprint formats such as maps or CD-ROMs. Adjust line 1a, i.e., last year's Volumes Held, and provide a footnote if you are adding records to the OPAC for government documents previously held but not counted as part of Volumes Held (line 1a).

Question 8. Computer files. Include the number of pieces of computer-readable disks, tapes, CD-ROMs, and similar machine-readable files comprising data or programs that are locally held as part of the library's collections available to library clients. Examples are U.S. Census data tapes, sample research software, locally-mounted databases, and reference tools on CD-ROM, tape or disk. Exclude bibliographic records used to manage the collection (i.e., the library's own catalog in machine-readable form), library system software, and microcomputer software used only by the library staff.

Question 9. Manuscripts and archives. Include both manuscripts and archives measured in linear feet.

Question 10. Cartographic materials. Include the numbers of pieces of two- and three-dimensional maps and globes. Include satellite and aerial photographs and images. 
Question 11. Graphic materials. Include the number of pieces of prints, pictures, photographs, postcards, slides, transparencies, film strips, and the like.

Question 12. Audio materials. Include the number of pieces of audiocassettes, phonodiscs, audio compact discs, reelto-reel tapes, and other sound recordings.

Question 13. Film and video materials. Include the number of pieces of motion pictures, videocassettes, video laser discs, and similar visual materials.

Questions 14-20. Expenditures. Report all expenditures of funds that come to the library from the regular institutional budget, and from sources such as research grants, special projects, gifts and endowments, and fees for service. (For question 17, include non-library funds; see instruction Q17.) Do not report encumbrances of funds that have not yet been expended. Canadian libraries should report expenditures in Canadian dollars. (For your information, if interested in determining figures in U.S. dollars, divide Canadian dollar amounts by 1.1323, the average monthly noon exchange rate published in the Bank of Canada Review for the period July 2006-June 2007.) Please round figures to the nearest dollar.

Question 15a. Monographs. Report expenditures for volumes purchased counted on line 2.

Question 15b. Serial titles. Report expenditures for serial titles counted on line 4a. Exclude unnumbered monographic and publishers' series, and encumbrances.

Question 15c. Other library materials. Include expenditures for all materials not reported in Questions 15a and 15b, e.g., backfiles of serials, charts and maps, audiovisual materials, manuscripts, etc. If expenditures for these materials are included in lines $15 \mathrm{a}$ and / or 15b and cannot be disaggregated, please report U/A and provide a footnote. Do not include encumbrances.

Question 15d. Miscellaneous expenditures. Include any other materials funds expenditures not included in questions 15a-c, e.g., expenditures for bibliographic utilities, literature searching, security devices, memberships for the purposes of publications, etc. Please list categories, with amounts, in a footnote. Note: If your library does not use materials funds for non-materials expenditures-i.e., if those expenditures are included in "Other Operating Expenditures"— report 0, not $\mathbf{U} / \mathbf{A}$, on line $15 \mathrm{~d}$.

Question 16. Contract Binding. Include only contract expenditures for binding done outside the library. If all binding is done in-house, state this fact and give in-house expenditures in a footnote; do not include personnel expenditures. (This figure should also be reported in the 2006-07 ARL Preservation Survey, question 7b.)

Questions 17. Salaries and wages. Exclude fringe benefits. If professional and support staff salaries cannot be separated, enter $\mathbf{U} / \mathbf{A}$, on lines $17 \mathrm{a}$ and $17 \mathrm{~b}$ and enter total staff on line 17 .

Question 17c. Salaries and wages: Student Assistants. Report 100\% of student wages regardless of budgetary source of funds. Include federal and local funds for work study students.

Question 19. Other operating expenditures. Exclude expenditures for buildings, maintenance, and fringe benefits.

Questions 21-25. Electronic expenditures. These items are intended to indicate what portion of your institution's total library expenditures are dedicated to electronic resources and services. Please use the Footnotes to indicate any 
electronic materials expenditures you believe not to be covered by these questions. Many expenditures recorded in these questions should have been included in Question 20, total library expenditures.

Question 21. One-time electronic resource purchases. Report expenditures that are not current serials (i.e. are nonsubscription, one-time, or monographic in nature) for software and machine-readable materials considered part of the collections. Examples include periodical backfiles, literature collections, one-time costs for JSTOR membership, etc. Expenditures reported here may be derived from any of the following categories: Monographs (Q15a), Other Library Materials (Q15c), Miscellaneous (Q15d), or Other Operating Expenditures (Q19).

Question 22. Ongoing electronic resource purchases. Report subscription expenditures (or those which are expected to be ongoing commitments) for serial publications whose primary format is electronic and for online searches of remote databases such as OCLC FirstSearch, DIALOG, Lexis-Nexis, etc. Examples include paid subscriptions for electronic journals and indexes / abstracts available via the Internet, CD-ROM serials, and annual access fees for resources purchased on a "one-time" basis, such as literature collections, JSTOR membership, etc. Not all items whose expenditures are counted here will be included in Serial titles currently received (Question 4) or Serials Expenditures (Question 15b).

Q23. Bibliographic Utilities, Networks, and Consortia. Because it is increasingly common for ARL Libraries to enter into consortial arrangements to purchase access to electronic resources, both "Library" and "External" expenditure blanks and instructions are provided. Please use afootnote to describe expenditures that you believe are not covered by the question, or situations that do not seem to fit the instructions.

Q23a. From internal library sources. Report expenditures paid by the Library for services provided by national, regional, and local bibliographic utilities, networks, and consortia, such as OCLC and RLG, unless for user database access and subscriptions, which should be reported in Questions 21 or 22. Include only expenditures that are part of Other Operating Expenditures (Q19).

Q23b. From external sources. If your library receives access to computer files, electronic serials or search services through one or more centrally-funded system or consortial arrangements for which it does not pay fully and/or directly (for example, funding is provided by the state on behalf of all members), enter the amount paid by external bodies on its behalf. If the specific dollar amount is not known, but the total student FTE for the consortium and amount spent for the academic members are known, divide the overall amount spent by your institution's share of the total student FTE.

Q24. Computer hardware and software. Report expenditures from the library budget for computer hardware and software used to support library operations, whether purchased or leased, mainframe or microcomputer, and whether for staff or public use. Include expenditures for: maintenance; equipment used to run information service products when those expenditures can be separated from the price of the product; telecommunications infrastructure costs, such as wiring, hubs, routers, etc. Include only expenditures that are part of Other Operating Expenditures (Q19).

Q25. Document Delivery/Interlibrary Loan. Report expenditures for document delivery and interlibrary loan services (both borrowing and lending). Include fees paid for photocopies, costs of telefacsimile transmission, royalties and access fees paid to provide document delivery or interlibrary loan. Include fees paid to bibliographic utilities if the portion paid for interlibrary loan can be separately counted. Include only expenditures that are part of Miscellaneous Materials Expenditures (Q15d) or Other Operating Expenditures (Q19), and only for those ILL/DD programs with data recorded in Questions 34-35.

Questions 26. Personnel. Report the number of FTE (full-time equivalent) staff in filled positions, or positions that are only temporarily vacant. ARL defines temporarily vacant positions as positions that were vacated during the fiscal year for which ARL data were submitted, for which there is a firm intent to refill, and for which there are expenditures for 
salaries reported on line 17.

Include cost recovery positions and staff hired for special projects and grants, but provide an explanatory footnote indicating the number of such staff. If such staff cannot be included, provide a footnote. To compute full-time equivalents of part-time employees and student assistants, take the total number of hours per week (or year) worked by part-time employees in each category and divide it by the number of hours considered by the reporting library to be a full-time work week (or year). Round figures to the nearest whole numbers.

Question 26a. Professional Staff. Since the criteria for determining professional status vary among libraries, there is no attempt to define the term "professional." Each library should report those staff members it considers professional, including, when appropriate, staff who are not librarians in the strict sense of the term, for example computer experts, systems analysts, or budget officers.

Question 26b. Support Staff. Report the total FTE (see instruction 26) of staff not included in 26a.

Question 26c. Student Assistants. Report the total FTE (see instruction Q26) of student assistants employed on an hourly basis whose wages are paid from funds under library control or from a budget other than the library's, including federal work-study programs. Exclude maintenance and custodial staff.

Question 27. Number of staffed library service points. Count the number of staffed public service points in the main library and in all branch libraries reported in this inventory, including reference desks, information desks, circulation, current periodicals, reserve rooms, reprographic services (if staffed as a public facility), etc. Report the number of designated locations, not the number of staff.

Question 28. Number of weekly public service hours. Report an unduplicated count of the total public service hours per typical full-service week (i.e., no holidays or other special accommodations) across both main library and branches using the following method (corresponds to IPEDS): If a library is open from 9:00 a.m. to 5:00 p.m. Monday through Friday, it should report 40 hours per week. If several of its branches are also open during these hours, the figure remains 40 hours per week. Should Branch A also be open one evening from 7:00 p.m. to 9:00 p.m., the total hours during which users can find service somewhere within the system becomes 42 hours per week. If Branch B is open the same hours on the same evening, the count is still 42, but if Branch B is open two hours on another evening, or remains open two hours later, the total is then 44 hours per week. Exclude 24-hour unstaffed reserve or similar reading rooms. The maximum total is 168 (i.e., a staffed reading room open 7 days per week, 24 hours per day).

Questions 29-30. Instruction. Sampling based on a typical week may be used to extrapolate TO A FULL YEAR for Questions 29 and 30. Please indicate if responses are based on sampling.

Question 29. Presentations to Groups. Report the total number of sessions during the year of presentations made as part of formal bibliographic instruction programs and through other planned class presentations, orientation sessions, and tours. If the library sponsors multi-session or credit courses that meet several times over the course of a semester, each session should be counted. Presentations to groups may be for either bibliographic instruction, cultural, recreational, or educational purposes. Presentations both on and off the premises should be included as long as they are sponsored by the library. Do not include meetings sponsored by other groups using library meeting rooms. Do not include training for library staff; the purpose of this question is to capture information about the services the library provides for its clientele. Please indicate if the figure is based on sampling.

Question 30. Participants in Group Presentations. Report the total number of participants in the presentations reported on line 29. For multi-session classes with a constant enrollment, count each person only once. Personal, one-to-one 
instruction in the use of sources should be counted as reference transactions on line 31. Please indicate if the figure is based on sampling. Use a footnote to describe any special situations.

Question 31. Reference Transactions. Report the total number of reference transactions. A reference transaction is an information contact that involves the knowledge, use, recommendations, interpretation, or instruction in the use of one or more information sources by a member of the library staff. The term includes information and referral service. Information sources include (a) printed and nonprinted material; (b) machine-readable databases (including computer-assisted instruction); (c) the library's own catalogs and other holdings records; (d) other libraries and institutions through communication or referral; and (e) persons both inside and outside the library. When a staff member uses information gained from previous use of information sources to answer a question, the transaction is reported as a reference transaction even if the source is not consulted again.

If a contact includes both reference and directional services, it should be reported as one reference transaction. Include virtual reference transactions (e.g., e-mail, WWW form, chat). Duration should not be an element in determining whether a transaction is a reference transaction. Sampling based on a typical week may be used to extrapolate TO A FULL YEAR for Question 31. Please indicate if the figure is based on sampling.

EXCLUDE SIMPLE DIRECTIONAL QUESTIONS. A directional transaction is an information contact that facilitates the logistical use of the library and that does not involve the knowledge, use, recommendations, interpretation, or instruction in the use of any information sources other than those that describe the library, such as schedules, floor plans, and handbooks.

Questions 32-33. Circulation. For Question 32, count the number of initial circulations during the fiscal year from the general collection for use usually (although not always) outside the library. Do not count renewals. Include circulations to and from remote storage facilities for library users (i.e., do not include transactions reflecting transfers or stages of technical processing). Count the total number of items lent, not the number of borrowers.

For Question 33, report total circulation for the fiscal year including initial transactions reported on line 32 and renewal transactions. Exclude reserve circulations; these are no longer reported.

Questions 34-35. Interlibrary Loans. Report the number of requests for material (both returnables and non-returnables) provided to other libraries on line 34 and the number of filled requests received from other libraries or providers on line 35. On both lines, include originals, photocopies, and materials sent by telefacsimile or other forms of electronic transmission. Include patron-initiated transactions. Exclude requests for materials locally owned and available on the shelves or electronically. Do not include transactions between libraries covered by this questionnaire.

Questions 36. Ph.D. Degrees. Report the number awarded during the 2006-07 fiscal year. Please note that only the number of $\underline{P h . D}$. degrees are to be counted. Statistics on all other advanced degrees (e.g., D.Ed., D.P.A., M.D., J.D.) should not be reported in this survey. If you are unable to provide a figure for Ph.D.s only, please add a footnote.

Question 37. Ph.D. Fields. For the purposes of this report, Ph.D. fields are defined as the specific discipline specialties enumerated in the U.S. Department of Education's Integrated Postsecondary Education Data System (IPEDS) "Completions" Survey. Although the IPEDS form requests figures for all doctoral degrees, only fields in which $\underline{\text { Ph.D.s }}$ are awarded should be reported on the ARL questionnaire. Any exceptions should be footnoted. 
Question 38. Instructional Faculty. Instructional faculty are defined by the U.S. Dept. of Education as

members of the instruction/research staff who are employed full-time as defined by the institution, including faculty with released time for research and faculty on sabbatical leave.

Full-time counts generally exclude faculty who are employed to teach fewer than two semesters, three quarters, two trimesters, or two four-month sessions; replacements for faculty on sabbatical leave or leave without pay; faculty for preclinical and clinical medicine; faculty who are donating their services; faculty who are members of military organizations and paid on a different pay scale from civilian employees; academic officers, whose primary duties are administrative; and graduate students who assist in the instruction of courses. Please be sure the number reported, and the basis for counting, are consistent with those for 2005-06 (unless in previous years faculty were counted who should have been excluded according to the above definition). Please footnote any discrepancies.

Questions 39-42. Enrollment. U.S. libraries should use the Fall 2006 enrollment figures reported to the Department of Education on the Integrated Postsecondary Education Data System survey. Please check these figures against the enrollment figures reported to ARL last year to ensure consistency and accuracy. Note: In the past, the number of parttime students reported was FTE; the number now reported to IPEDS is a head count of part-time students. Canadian libraries should note that the category "graduate students" as reported here includes all post-baccalaureate students.

\section{FOOTNOTES}

Please consult the data entry Web interface (www.arlstatistics.org) for a copy of last year's footnotes. These can be found under "Historical Data" by clicking a survey's arrow icon under the "View Summary" column and then selecting the "Footnotes" tab. Explanatory footnotes will be included with the published statistics. Reporting libraries are urged to record in the footnote section any information that would clarify the figures submitted, e.g., the inclusion and exclusion of branch campus libraries (see the "General Instructions" for definition of branch campus libraries). Please make an effort to word your footnotes in a manner consistent with notes appearing in the published report, so that the ARL Office can interpret your footnotes correctly.

Submit the completed questionnaire

by January 30, 2008.

For assistance, please e-mail Martha Kyrillidou (martha@arl.org) or Mark Young (stats-ra@arl.org)

Tel. (202) 296-2296.

40 · ARL Academic Law Library Statistics 2006-2007 
ARL Statistics 2006-2007

WORKSHEET

This worksheet is designed to help you plan your submission for the 2006-07 ARL Statistics. The figures on this worksheet should be similar to those in the "Summary" page of your web form, except in cases where data are unavailable. If an exact figure is unavailable, use "NA/UA". If the appropriate answer is zero or none, use "0."

Reporting Institution Date Returned to ARL

Report Prepared by (name)

Title

E-mail address Phone number

Contact person (if different)

Title

E-mail address

Phone number

PAGE ONE - VOLUMES

1. Volumes held June 30, $2007(1 a+1 b)$

(1)

1a. Volumes held June 30, 2006

(1a)

1b. Volumes added during the year (1b.i - 1b.ii) (1b)

(i) Volumes added - Gross

(1b.i)

(ii) Volumes withdrawn during year

(1b.ii)

2. Number of monographic volumes purchased

(2)

3. Basis of volume count is:

(3) Physical Bibliographic 
PAGE TWO - OTHER COLLECTIONS

SERIALS

4. Total number of serial titles currently received, including periodicals $(4 a+4 b)$

(4)

4a. Number of serial titles currently purchased

(4a)

4a.i Electronic

(4a.i)

4a.ii Print (and other format) serials purchased (4a.ii)

4b. Number of serial titles currently received but not purchased

$(4 b)$

4b.i Electronic

4b.ii Print (and other format) serial received but not purchased

(Exchanges, gifts, deposits, etc. See instructions.) (4b.i)

(4b.ii) 
PAGE THREE - EXPENDITURES

14. Are the below figures reported in Canadian dollars?

Yes No

15. Total Library Materials Expenditures $(15 a+15 b+15 c+15 d)$

15a. Monographs

15b. Serial titles, including periodicals

15c. Other Library Materials

15d. Miscellaneous

16. Contract binding

17. Total Salaries and Wages $(17 a+17 b+17 c)$

17a. Professional staff

17b. Support staff

17c. Student assistants (17a)

(15a)

(16)

18. Fringe benefits are included in expenditures for salaries and wages? (18) Yes No

19. Other operating expenditures

20. Total library expenditures

$$
(15+16+17+19)
$$

\section{ELECTRONIC MATERIALS EXPENDITURES}

21. One-time electronic resource purchases

22. Ongoing electronic resource purchases (e.g., subscriptions, annual license fees)

23. Bibliographic Utilities, Networks, and Consortia

23a. From internal library sources

23b. From external sources

24. Computer hardware and software

25. Document Delivery/Interlibrary Loan (23a)

(23b)

(24)

(25) 
PERSONNEL (Round figures to nearest whole number.)

26. Total Staff FTE $(26 a+26 b+26 c)$

26a. Professional staff, FTE

26b. Support staff, FTE

26c. Student assistants, FTE (26a)

(26b)

$(26 c)$

\section{STAFFED SERVICE POINTS AND HOURS}

27. Number of staffed library service points

(27)

28. Number of weekly public service hours

\section{INSTRUCTION}

29. Number of library presentations to groups

29a. Is the library presentations figure based on sampling?

(29a) Yes No

30. Number of total participants in group presentations reported in line 29

30a. Is the total participants in group presentations Yes No figure based on sampling?

\section{REFERENCE}

31. Number of reference transactions

31a. Is the reference transactions figure based on sampling?

(31a) Yes No 


\section{CIRCULATION}

32. Number of initial circulations (excluding reserves)

33. Total circulations (initial and renewals, excluding reserves)

\section{INTERLIBRARY LOANS}

34. Total number of filled requests provided to other libraries

35. Total number of filled requests received from other libraries or providers

\section{Ph.D. DEGREES AND FACULTY}

36. Number of Ph.D.s awarded in FY2006-07

37. Number of fields in which Ph.D.s can be awarded

38. Number of full-time instructional faculty in FY2006-07

ENROLLMENT - FALL 2006

(Line numbers refer to IPEDS survey form.)

39. Full-time students, undergraduate and graduate

(Add line 8, columns $15 \mathcal{E} 16$, and line 14, columns 15 \& 16.)

40. Part-time students, undergraduate and graduate

(Add line 22, columns $15 \& 16$, and line 28, columns 15 \& 16.)

42. Part-time graduate students (Line 28, columns 15 \& 16.)

\section{FOOTNOTES}

On the web form, you will be able to add footnotes to individual questions, as well as footnotes that apply to your entire institution. Please provide any information which would clarify the figures submitted, e.g., the inclusion of branch campus libraries or any special projects which might cause radical increases or decreases. Please use the footnotes in the ARL Statistics 2005-2006 for comparison if necessary. Please consult the data entry Web interface (www.arlstatistics.org) for a copy of last year's footnotes. These can be found under "Historical Data" by clicking a 
survey's arrow icon under the "View Summary" column and then selecting the "Footnotes" tab. Please make an effort to word your footnotes in a manner consistent with notes appearing in the published report, so that the ARL Office can interpret your footnotes correctly.

Submit the completed questionnaire by January 30, 2008.

For assistance, please e-mail Martha Kyrillidou (martha@arl.org) or Mark Young (stats-ra@arl.org) Tel. (202) 296-2296. 
Footnotes to the ARL Academic Law Library Statistics 2006-2007

Footnotes may also include errata and corrections to data not previously reported from prior years. Numbers in parentheses refer to columns in Library Data Tables and to Questionnaire numbers. Unless otherwise noted, all figures are as of $06 / 30 / 2007$.

\begin{tabular}{|c|c|}
\hline $\begin{array}{l}\text { QUESTION } \\
\text { NUMBER }\end{array}$ & FOOTNOTE \\
\hline \multicolumn{2}{|l|}{ ALABAMA } \\
\hline & Figures are as of $09 / 30 / 2007$. \\
\hline 19 & Includes bibliographic utilities and literature searching. \\
\hline \multicolumn{2}{|l|}{ ARIZONA } \\
\hline 1b.ii & Library is undergoing facility renovation and numerous volumes were discarded as a result. \\
\hline 2 & Monographic titles purchased are only counted. \\
\hline \multicolumn{2}{|l|}{ BOSTON } \\
\hline $1 \mathrm{a}$ & Volumes held June 30, 2006 revised to 327,976. \\
\hline \multicolumn{2}{|c|}{ BOSTON COLLEGE } \\
\hline $1 \mathrm{a}$ & $\begin{array}{l}\text { Volumes held June } 30,2006 \text { revised to } 240,957 \text {. This number has been adjusted as per the instructions for (1b). The adjustment reflects } \\
\text { the addition of 2,795 cataloged e-book titles which are part of digital products reported in the footnote for ( } 2 \text { ). In future years we will } \\
\text { add only additional cataloged e-books in this product to (1bi). }\end{array}$ \\
\hline 2 & $\begin{array}{l}\text { This figure includes 2,795 e-books included in the following digital products: HeinOnline: 1,581; LLMC Digital (Law Library } \\
\text { Microform Consortium): } 977 \text {; BNA-ALL (Bureau of National Affairs): } 237 .\end{array}$ \\
\hline $4-4 a$ & See footnotes for Questions 4a.i. and 4a.ii. \\
\hline 4a.i & These numbers reflect partial (not complete) de-duping of e-resources between the University and Law Libraries. \\
\hline 4a.ii & $\begin{array}{l}\text { Unable to de-dupe law library print resources against either law library e-resources or against University library print or e-resources } \\
\text { this year. }\end{array}$ \\
\hline 4b-4b.ii & Not de-duped. \\
\hline
\end{tabular}

\section{BRIGHAM YOUNG}

Figures are as of $12 / 31 / 2006$.

13 This figure is very low and included with the Audio count.

\section{CALIFORNIA, DAVIS}

4a, 4b Number was NA/UA for 2005-2006.

6-12 No longer useful units withdrawn causing minor drop from 2005-2006 total.

$34 \quad$ Change in filled requests given low total number is not statistically significant.

36-42 Institutional-level data reported in ARL Statistics.

\section{CALIFORNIA, LOS ANGELES}

4a.i Unable to breakout for Law only their electronic resources from total electronic serials.

\section{CASE WESTERN RESERVE}

4a.i The law library also has access to 37,913 e-journals that are reported in the main library's statistics.

15d OCLC expenditures.

17, 18, 26 Expenditures and staff FTE have decreased since the IT department for the Law Library no longer reports to the Director of the Law Library.

\section{CHICAGO}

$1,1 \mathrm{~b} \quad$ Withdrew 38,608 volumes that were duplicative or available in electronic form and in the university library.

$2 \quad$ Reflects receipt of books ordered late in 2005-2006 and a new policy to expend all book funds before 7/07.

13 Established a popular DVD collection and purchased 409 DVDs.

15-15a In 2006-2007, electronic expenditures data were excluded. The increase in monographic expenditures was due to a new policy to reduce year-end commitments and expend book funds before 7/07.

15b In 2005-2006, electronic expenditures data were excluded. The correct amount for (15b) for 2005-2006 was $\$ 1,327,931$.

15c The 2005-2006 survey incorrectly reported (15c) as $\$ 293,461$. The correct figure for other library materials (15c) was $\$ 18,028$.

17c No student project staff were employed in 2006-2007.

21 The correct amount for (21) for 2005-2006 was $\$ 409,974$.

26-26b No project staff were employed in 2006-2007.

The library was undergoing a renovation all year and 200,000 volumes were placed in dark storage.

34 Number of titles lent varies based on the unpredictable requests from other libraries; further, 200,000 volumes in storage were unavailable for ILL.

35 May be attributed to the need to borrow titles owned, but now in dark storage. 
Excludes the Raymond Walters College and Clermont College libraries.

4a.i It was too difficult to separate our e-journals from the other jurisdictions within the university. We are reporting only our paper titles, allowing the e-journals to be counted in the "main" count.

\section{COLUMBIA}

1a Volumes held June 30, 2006 revised to 892,389. Restated from 2005-2006 to include 21,811 e-volumes.

4a.i Includes Hein Online and LLMC (de-duped from paper total).

24 Includes expenditures for computer maintenance.

28 Number included in Butler count (102 for Law Library only, 107 total for Columbia).

$34 \quad$ Filled requests provided to other libraries includes 4,888 from fee-based service.

CORNELL

Volumes held June 30, 2006 revised to 548,518. Change from ending figure for 2005-2006 reflects the fact that additional e-books were counted. E-book counts are as of $10 / 2007$.

1b.i Includes a small number of manually cataloged e-books.

Estimate only. Divisions by paid/ unpaid unavailable. $21 \%$ of the titles in this count were estimated to be print. 3,300 of these titles were estimated to be non-current e-titles. This count was made particularly difficult by the fact that online records do not exist for two of the Law Library's largest e-journal packages as they are not available beyond the Law School (these packages were not counted in the past). For the largest aggregators, estimated percentages used to de-dupe counts were percentages available for central and Law online records combined. The central estimate to de-dupe between print and e was arrived at through manually searching a sample. Print counts include numbered and unnumbered monographic series and non-serial continuations. Decrease from 2005-2006 due in large part to a new sampling method instituted at CUL.

DUKE

$1 \mathrm{~b} \quad$ Volumes showed negative growth because a large number of duplicate titles were discarded in preparation for library renovation. The renovated space resulted in a $20 \%$ loss of shelf space.

EMORY

The Law Library has recently removed a large quantity of its volumes, primarily those whose content is now available online. For this reason, this value is a negative figure, representing a reduction in size.

$2 \quad$ Fewer funds are available for purchase of new titles due to large amount of operational budget going toward continuation costs.

10 Three of eight maps have been discarded. The size of the item count is small, so a loss of three maps constitutes a large percentage difference from 2005-2006.

13 A large quantity of videos were removed from collection due to loss of functional capacity.

\section{FLORIDA}

1a Volumes held June 30, 2006 revised to 319,955.

$5 \quad$ Not all government documents are included in this count. Some serial government documents are added to our LC classified collection and get counted at time of processing. Others serial government documents are added to the documents collection and are included in (7).

\section{FLORIDA STATE}

1a Volumes held June 30, 2006 revised to 304,954.

39, 41 Information from internal sources. IPEDS was unavailable.

\section{GEORGE WASHINGTON}

34-35 ILL statistics for 2005-2006 were unusually low for unknown reasons. Statistics for 2006-2007 returned to normal levels.

\section{GEORGETOWN}

29-30 Data for 2006-2007 reflects an expanded teaching presence as well as improved tracking and reporting over previous year.

\section{GEORGIA}

Branch library numbers have been included in the Main Library statistics (see ARL Statistics).

1a Volumes held June 30, 2006 revised to 614,121. Number adjusted to include e-books.

2 Includes purchase of 118,210 Eighteen Century Collection Online.

HARVARD

1b.ii Increase due to de-accessions.

15-21 Increase in endowment allocation for 2006-2007.

HOUSTON

1a Volumes held June 30, 2006 revised to 533,721. 2005-2006 number (260,684) was reported incorrectly.

$1 \mathrm{~b} \quad$ A large weeding project was undertaken during the year.

\section{HOWARD}

$6 \quad$ Processing of 5-year backlog.

\section{ILLINOIS-URBANA}

This figure only includes (15) and (19). This does not include (16). 
IOWA

2, 4a, 4b Estimate.

17a Does not include director's salary.

KANSAS

1b.i We changed how we counted volumes to reflect new ABA standards. We had previously included cataloged electronic serial title volumes that are now counted differently. We adjusted to remove electronic volumes previously reported and to add electronic volumes counted with new ABA standards.

1b.ii We withdrew 40,256 volumes to accommodate library remodeling.

$2 \quad$ Includes electronic monographic volumes purchased in MOML and Supreme Court Records and Briefs.

4a Our reported number of current serials purchased dropped because of de-duping with Main and Medical libraries and because of cancellations in preparation for library remodeling. Number of current serials received but not purchased are included. Government document titles counted here.

10 Cartographic materials reflects number purchased.

12 Audio reflects number purchased.

26b One support staff position was converted to tenure track librarian position.

\section{LOUISIANA STATE}

1a Volumes held June 30, 2006 revised to 443,204. Includes 994 e-books not counted in 2005-2006.

1b.i Two of our electronic vendors, LLMC-Digital and Hein Online, added 3,610 e-books in the past year. In addition, our normal level of print volume purchases increased due to the purchase of several new state statute sets and the reinstatements and replacement of large sets following the Katrina-related cancellations.

15a Reflects the additional costs associated with the purchase of the additional state statutes, reinstatements, and replacements mentioned in question (1bi) above.

$15 \mathrm{~b} \quad$ Part of this increase was again due to the reinstatement of serial titles cancelled following Katrina. Much of the remainder was due to the change in language to (4a) which allowed us this year to count full-text electronic serial titles. In 2005-2006, these titles were excluded from the count and instead were reflected in the Supplementary Statistics. Since we could count these electronic serial titles this year, we included the cost to purchase access in the serial titles expenditures. In 2005-2006, we reflected these expenditures in Other library expenditures, (15c) (see below).

15c Excludes the cost of electronic serial titles that are instead included in (15b) (see above). The remaining amount reflects the expenditures for electronic database access but which cannot be tied to the cost of any particular electronic serial title as required to be reported in (15b).

19 2005-2006 amount included equipment expenditures which did not occur this year.

22 In 2005-2006, this question focused only on the cost of electronic serials which were excluded from inclusion by the language of (4a). This year's language is broader and allows us to report the cost of all ongoing electronic resource purchases.

24 In 2005-2006, we had no new computer purchases for the library, but we overlooked the cost of support for our computer system and so we underreported. This year's figure more accurately reflects those support costs, primarily telecommunications expenses.

MIAMI

17a Excludes law library director's salary.

36-42 Institutional-level data reported in ARL Statistics.

MICHIGAN

15d ILL, \$378 and mass de-acidification, \$50,717.

32-33 Includes reserves.

\section{MICHIGAN STATE}

4 Because of the ARL decision to count individual serial titles rather than serial subscriptions, some electronic sources have been counted by the individual titles they contain instead of counted as one subscription. In addition, since the last fiscal year more of our serial titles have been represented by bibliographic records in the catalog.

25 We have seen an increase in this budget line in both the number of transactions and the cost per transaction.

\section{MINNESOTA}

1a $\quad$ Adjusted to count volumes transferred from University Libraries.

$4 \mathrm{a}, 4 \mathrm{~b} \quad$ Breakdown of unique titles by branch library is not feasible.

11 Breakdown of A/V media holdings not entered because not available for main ARL statistics. Law Library holdings are Graphic, 13 Audio, 250, and Video 875

36 Breakdown corresponding to branch library not available. Counts for entire campus entered in main statistics.

\section{MISSOURI}


15-25 Expenditures as reported in Canadian dollars: (15) \$793,152; (15a) \$121,869; (15b) \$671,283; (15c) \$0; (15d) \$0;

(16) $\$ 8,532$; (17) \$765,241; (17a) \$334,832; (17b) \$430,409; (17c) \$0; (19) \$13,387; (20) \$1,580,312; (21) UA/NA; (22) \$134,282; (23a) UA/ NA; (23b) UA/NA; (24) UA/NA; (25) UA/NA.

\section{NEBRASKA}

4a.i Data for (4ai) and (4aii) unavailable for Law Library.

7,8,9 Figure unavailable.

\section{NEW YORK}

All figures as of $08 / 31 / 2007$.

1a Volumes held June 30, 2006 revised to 787,815.

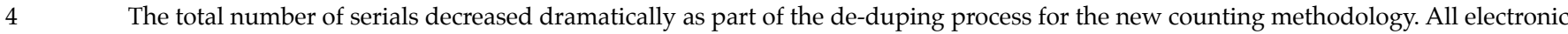
serials will only be reported through the Division of Libraries. The actual number of serial titles available from the Law Library is 8,268 .

4a.i Electronic serials totals for NYU are only being counted in the main Division of Libraries total. The actual number of purchased electronic serials at the Law Library is 118.

4b.i Electronic serials totals for NYU are only being counted in the main Division of Libraries total. The actual number of free electronic serials for the Law Library is 3,104.

36-42 PhD degrees, faculty, and enrollment numbers are all included in the Division of Libraries total.

\section{NORTH CAROLINA}

1a Volumes held June 30, 2006 revised to 326,308. Added 231 e-books not previously counted.

1b Added Making of Modern Law, containing 22,007 electronic books.

26 Library has 12.50 FTE professional staff, 25.5 FTE total.

\section{NOTRE DAME}

15d Certain expenditures that were included in miscellaneous expenditures in 2005-2006 have been reclassified to serials and other expenditures in 2006-2007. In addition, spending was reduced in 2006-2007 as compared to 2005-2006 levels.

\section{OHIO STATE}

4b All Ohio State libraries have cataloged access to 3,285 free electronic serials.

$23 \mathrm{~b} \quad$ Inseparable from total university's figure of $\$ 1,142,832$.

\section{OREGON}

$$
\text { Includes the Jaqua Law Library only. }
$$

1b.ii Weeding project.

22 One large payment was delayed and is now on a new payment schedule.

25 Unable to separate out law from general library ILL statistics.

\section{PENNSYLVANIA}

1a Volumes held June 30, 2006 revised to 617,298.

\section{PENNSYLVANIA STATE}

Included: Dickinson School of Law Library, and the H. Laddie Montague, Jr. Law Library branches: 104 Beam Building, University Park, PA 16802, 150 S. College Street, Carlisle, PA 17013.

1b.i E-books (counted as titles because volume count unavailable) = 27,598 titles. E-book Products Added: The making of Modern Law; LLMC Digital; BePress Journals; Hein Online; BNA All; CCH; Tax Management; U.S. Supreme Court records \& Briefs (Gale); U.S. Congressional Hearings (LexisNexis).

2 We purchased 25,728 monographic e-books this year, which were funded through our electronic resource purchases (22).

12-13 As part of our ongoing collection relocation, we drastically weeded our audio and video collections. Most of the titles counted last year were non-commercial, locally created tapes that have now been removed from the collection.

\section{QUEENS}

(36-37) and (39-42) as at November 2006, (38) as at October 2006. (4), (23), and (24) statistics included in Main Library Questionnaire. The numbers cannot be broken out.

15-25 Expenditures as reported in Canadian dollars: (15) \$698,835; (15a) \$293,551; (15b) \$401,650; (15c) \$3,634; (15d) UA/ NA; (16) $\$ 14,520$; (17) \$472,216; (17a) \$144,827; (17b) \$286,095; (17c) \$41,294; (19) \$24,905; (20) \$1,210,476; (21) UA/NA; (22) \$280,203; (23a) UA/NA; (23b) UA/NA; (24) UA/NA; (25) UA/NA.

\section{RUTGERS, CAMDEN}

12 Camden Law reported 10 Audio. Entered as 0 since Main Library can only report total Audio Visual materials.

13 Camden Law reported 76 Film \& Video. Entered as 0 since Main Library can only report total Audio Visual materials.

\section{RUTGERS, NEWARK}

1b.i-2 Includes 24,921 e-books: Making of Modern Law Legal Treaties.

12 Newark Law reported 858 Audio. Entered as 0 since Main Library can only report total Audio Visual materials.

13 Newark Law reported 309 Film \& Video. Entered as 0 since Main Library can only report total Audio Visual materials. 
15b, 15d 2005-2006 included database subscriptions $(\$ 100,812)$ in questions $(15 d)$; should have been included in question $15 b$ Serials titles including periodicals. 2006-07 included $\$ 140,899$ for database subscriptions in (15b).

17a 2005-2006 figure corrected to $\$ 583,550$ (Total Salaries \& Wages remains the same; correction is in distribution to Professional and Support staff).

17b 2005-2006 figure corrected to $\$ 475,887$ (Total Salaries \& Wages remains the same; correction is in distribution to Professional and Support staff).

\section{SASKATCHEWAN}

15-25 Expenditures as reported in Canadian dollars: (15) \$861,448; (15a) \$117,224; (15b) \$744,224; (15c) UA/ NA; (15d) UA/ NA; (16) \$7,139; (17) \$416,073; (17a) \$209,991; (17b) \$183,656; (17c) \$22,426; (19) UA/NA; (20) \$1,284,660; (21) UA/NA; (22) UA/NA; (23a) UA/NA; (23b) UA/NA; (24) UA/NA; (25) UA/NA.

15c Expenditures included in (16).

32 2006-2007 data much higher than the 2005-2006 data due to a revision in reporting process. The 2005-2006 data would read 13,463 instead of 7,964 under new reporting process.

33 2006-2007 data much higher than the 2005-2006 data due to a revision in reporting process. The 2005-2006 data would read 17,142 instead of 11,623 under new reporting process.

\section{SUNY-BUFFALO}

1a Volumes held June 30, 2006 revised to 298,149.

4a.i, 4b.i Electronic serials are reported on the Main Library summary for the entire Library System.

15d Westlaw and LexisNexis costs are reported here.

24 Expenses for this category are all included on the Main Library page.

32-33 The numbers reported are more accurate that those in past years due to the implementation of a new online system.

36-42 This question is answered on the main summary with institutional data.

TEMPLE

4a Number reflects de-duplication.

\section{TENNESSEE}

4a Law Titles Purchased: Electronic 669 [of original 1,247] (4a.i); Print 1,445 [of original 1,575] (4a.ii); Total 2,114 [of original 2,822] (4a).

4b Law Titles Not Purchased -- all NA/UA

28 UT Law Library is open 112 hours per week but occurrence of hours is within that of Main.

39-42 Included in Main total.

TORONTO

1a Volumes held June 30, 2006 revised to 206,331.

15-25 Expenditures as reported in Canadian dollars: (15) \$842,408; (15a) \$70,033; (15b) \$748,550; (15c) \$23,825; (15d) UA/ NA; (16) $\$ 12,951$; (17) \$1,180,080; (17a) \$712,226; (17b) \$400,144; (17c) \$67,710; (19) \$119,366; (20) \$2,154,805; (21) UA/NA; (22) \$83,717; (23a) UA/NA; (23b) UA/NA; (24) \$77,500; (25) UA/NA.

\section{TULANE}

1a Volumes held June 30, 2006 revised to 308,875.

$4 \quad$ Only aggregate serial data available, 5,977 serials overall.

15c Cannot be disaggregated.

\section{VANDERBILT}

17c Federal funds for work study students have been added to this total for the first time.

18 Fringe benefits are included.

\section{VIRGINIA}

Excludes: Health Sciences, Darden Graduate Business, Alderman, Astronomy, Biology/Psychology, Chemistry, Clemons Undergraduate, Education, Fiske Kimball Fine Arts, Math, Music, Physics, Brown Science \& Engineering, Small Special Collections libraries.

1a Volumes held June 30, 2006 revised to 652,884. Includes 21,812 e-books not included in previous surveys, this is the number of e-books held as of 6/30/07. We could not determine how many we held as of 6/30/06.

1b.i This number represents print volumes only.

1b.ii Law conducted a massive weeding project during 2006-2007.

2 There were no e-book purchases in 2006-2007.

$4 \quad$ Serials for all libraries are reported on the University Library (main) survey only.

15a Expenditures for print monographs only. No new e-books purchased.

18 Fringe benefits are included.

\section{WASHINGTON U.-ST. Louis}

1-1a Volumes held June 30, 2006 revised to 442,784. Includes 31,083 electronic books not previously reported. 
WESTERN ONTARIO

1b-1b.i 2005-2006 count included previously held items that were newly linked as part of a project as well as new items. 2006-2007 count includes new items.

4a.i, 4b.i Electronic serials for Law are included with the Main Library counts. These serials are available in all libraries.

Expenditures as reported in Canadian dollars: (15) \$810,005; (15a) \$120,847; (15b) \$689,158; (15c) UA/NA; (15d) \$0; (16) $\$ 13,084$; (17) \$348,811; (17a) \$213,091; (17b) \$125,045; (17c) \$10,675; (19) \$10,829; (20) \$1,182,729; (21) UA/NA; (22) UA/NA; (23a) UA/NA; (23b) \$0; (24) UA/NA; (25) \$0.

21-22 Included with Main Library. Purchase of electronic resources handled centrally and resources available to all libraries.

23a, 24 Centrally funded for all libraries at Western Libraries.

35 A central ILL office at Western Libraries handles filled requests received from other libraries or providers.

YALE

Volumes held June 30, 2006 revised to 876,997.

Fringe benefits are included. 
Appendix: ARL Member Libraries as of June 1, 2008

\begin{tabular}{|c|c|c|c|}
\hline Institution & Category & Full Name of Institution & Location \\
\hline Alabama & S & University of Alabama & Tuscaloosa, Alabama \\
\hline Alberta & $\mathrm{C}$ & University of Alberta & Edmonton, Alberta \\
\hline Arizona & S & University of Arizona & Tucson, Arizona \\
\hline Arizona State & S & Arizona State University & Tempe, Arizona \\
\hline Auburn & S & $\begin{array}{l}\text { Arizona tate University } \\
\text { Auburn University }\end{array}$ & Auburn, Alabama \\
\hline Boston & $\begin{array}{l}\mathrm{b} \\
\mathrm{b}\end{array}$ & Boston University & Boston, Massachusetts \\
\hline Boston College & $\begin{array}{l}P \\
b\end{array}$ & Boston College & Boston, Massachusetts \\
\hline Brigham Young & $\begin{array}{l}1 \\
P\end{array}$ & Brigham Young University & Provo, Utah \\
\hline British Columbia & $\mathrm{C}$ & University of British Columbia & Vancouver, British Columbia \\
\hline Brown & $\mathrm{P}$ & Brown University & Providence, Rhode Island \\
\hline Berkeley, California & S & University of California, Berkeley & California, Berkeley \\
\hline California, Davis & S & University of California, Davis & Davis, California \\
\hline California, Irvine & S & University of California, Irvine & Irvine, California \\
\hline California, Los Angeles & S & University of California, Los Angeles & Los Angeles, California \\
\hline California, Riverside & $\begin{array}{l}S \\
S\end{array}$ & University of California, Riverside & Riverside, California \\
\hline California, San Diego & $S$ & University of California, San Diego & La Jolla, California \\
\hline California, Santa Barbara & S & University of California, Santa Barbara & Santa Barbara, California \\
\hline Case Western Reserve & $\breve{P}$ & Case Western Reserve University & Cleveland, Ohio \\
\hline Chicago & $\mathrm{P}$ & University of Chicago & Chicago, Illinois \\
\hline Cincinnati & S & University of Cincinnati & Cincinnati, Ohio \\
\hline Colorado & S & University of Colorado & Boulder, Colorado \\
\hline Colorado State & S & Colorado State University & Fort Collins, Colorado \\
\hline Columbia & $\mathrm{P}$ & Columbia University & New York, New York \\
\hline Connecticut & S & University of Connecticut & Storrs, Connecticut \\
\hline Cornell & $\mathrm{P}$ & Cornell University & Ithaca, New York \\
\hline Dartmouth & $\mathrm{P}$ & Dartmouth College & Hanover, New Hampshire \\
\hline Delaware & S & University of Delaware & Newark, Delaware \\
\hline Duke & $\mathrm{P}$ & Duke University & Durham, North Carolina \\
\hline Emory & $\mathrm{P}$ & Emory University & Atlanta, Georgia \\
\hline Florida & S & $\begin{array}{l}\text { Emory University } \\
\text { University of Florida }\end{array}$ & Gainesville, Florida \\
\hline Florida State & S & & Tallahassee, Florida \\
\hline George Washington & $\mathrm{P}$ & George Washington University & Washington, DC \\
\hline Georgetown & $P$ & Georgetown University & Washington, DC \\
\hline Georgia & S & University of Georgia & Athens, Georgia \\
\hline Georgia Tech & S & Georgia Institute of Technology & Atlanta, Georgia \\
\hline Guelph & $\mathrm{C}$ & University of Guelph & Guelph, Ontario \\
\hline Harvard & $\mathrm{P}$ & Harvard University & Cambridge, Massachusetts \\
\hline Hawaii & S & University of Hawaii & Honolulu, Hawaii \\
\hline Houston & S & & Houston, Texas \\
\hline Howard & $\mathrm{P}$ & Howard University & Washington, DC \\
\hline Illinois, Chicago & S & $\begin{array}{l}\text { Howara University } \\
\text { University of Illinois at Chicago }\end{array}$ & Chicago, Illinois \\
\hline Illinois, Urbana & S & University of Illinois at Cnicago & Urbana, Illinois \\
\hline Indiana & $S$ & $\begin{array}{l}\text { Indiana University } \\
\text { Intona }\end{array}$ & Bloomington, Indiana \\
\hline Iowa & S & University of Iowa & Iowa City, Iowa \\
\hline Iowa State & S & Iowa State University & Ames, Iowa \\
\hline Johns Hopkins & $\mathrm{P}$ & Johns Hopkins University & Baltimore, Maryland \\
\hline Kansas & S & University of Kansas & Lawrence, Kansas \\
\hline Kent State & S & Kent State University & Kent, Ohio \\
\hline Kentucky & $S$ & University of Kentucky & Lexington, Kentucky \\
\hline Laval & $\mathrm{C}$ & Laval University & Quebec, Quebec \\
\hline Louisiana State & S & Louisiana State University & Baton Rouge, Louisiana \\
\hline Louisville & S & University of Louisville & Louisville, Kentucky \\
\hline McGill & $\mathrm{C}$ & McGill University & Montreal, Quebec \\
\hline McMaster & $\mathrm{C}$ & McMaster University & Hamilton, Ontario \\
\hline Manitoba & $\mathrm{C}$ & University of Manitoba & Winnipeg, Manitoba \\
\hline Maryland & S & University of Maryland & College Park, Maryland \\
\hline Massachusetts & S & University of Massachusetts & Amherst, Massachusetts \\
\hline MIT & $\mathrm{P}$ & Massachusetts Institute of Technology & Cambridge, Massachusetts \\
\hline Miami & $\mathrm{P}$ & University of Miami & Coral Gables, Florida \\
\hline Michigan & S & University of Michigan & Ann Arbor, Michigan \\
\hline Michigan State & S & $\begin{array}{l}\text { University or Micnigan } \\
\text { Michigan State University }\end{array}$ & East Lansing, Michigan \\
\hline Minnesota & S & University of Minnesota & Minneapolis, Minnesota \\
\hline
\end{tabular}




\begin{tabular}{|c|c|c|c|}
\hline Missouri & $\mathrm{S}$ & University of Missouri & Columbia, Missouri \\
\hline Montreal & $\mathrm{C}$ & University of Montreal & Montreal, Quebec \\
\hline Nebraska & $S$ & University of Nebraska-Lincoln & Lincoln, Nebraska \\
\hline New Mexico & $\mathrm{S}$ & University of New Mexico & Albuquerque, New Mexico \\
\hline New York & $\mathrm{P}$ & New York University & New York, New York \\
\hline North Carolina & $\mathrm{S}$ & University of North Carolina & Chapel Hill, North Carolina \\
\hline North Carolina State & S & North Carolina State University & Raleigh, North Carolina \\
\hline Northwestern & $\mathrm{P}$ & Northwestern University & Evanston, Illinois \\
\hline Notre Dame & $\mathrm{P}$ & University of Notre Dame & Notre Dame, Indiana \\
\hline Ohio & $\mathrm{S}$ & Ohio University & Athens, Ohio \\
\hline Ohio State & $\mathrm{S}$ & Ohio State University & Columbus, Ohio \\
\hline Oklahoma & $\mathrm{S}$ & University of Oklahoma & Norman, Oklahoma \\
\hline Oklahoma State & S & Oklahoma State University & Stillwater, Oklahoma \\
\hline Oregon & $\mathrm{S}$ & University of Oregon & Eugene, Oregon \\
\hline Pennsylvania & $\mathrm{P}$ & University of Pennsylvania & Philadelphia, Pennsylvania \\
\hline Pennsylvania State & $\mathrm{S}$ & Pennsylvania State University & University Park, Pennsylvania \\
\hline Pittsburgh & $\mathrm{S}$ & University of Pittsburgh & Pittsburgh, Pennsylvania \\
\hline Princeton & $\mathrm{P}$ & Princeton University & Princeton, New Jersey \\
\hline Purdue & $S$ & Purdue University & West Lafayette, Indiana \\
\hline Queen's & $\mathrm{C}$ & Queen's University & Kingston, Ontario \\
\hline Rice & $\mathrm{P}$ & Rice University & Houston, Texas \\
\hline Rochester & $\mathrm{P}$ & University of Rochester & Rochester, New York \\
\hline Rutgers & S & Rutgers University & New Brunswick, New Jersey \\
\hline Saskatchewan & $\mathrm{C}$ & University of Saskatchewan & Saskatoon, Saskatchewan \\
\hline South Carolina & $\mathrm{S}$ & University of South Carolina & Columbia, South Carolina \\
\hline Southern California & $\mathrm{P}$ & University of Southern California & Los Angeles, California \\
\hline Southern Illinois & $\mathrm{S}$ & Southern Illinois University & Carbondale, Illinois \\
\hline SUNY-Albany & $\mathrm{S}$ & & Albany, New York \\
\hline SUNY-Buffalo & $\mathrm{S}$ & University at Buffalo, State Univ. of New York & Buffalo, New York \\
\hline SUNY-Stony Brook & S & State University of New York at Stony Brook & Stony Brook, New York \\
\hline Syracuse & $\mathrm{P}$ & Syracuse University & Syracuse, New York \\
\hline Temple & $\mathrm{S}$ & Temple University & Philadelphia, Pennsylvania \\
\hline Tennessee & S & University of Tennessee & Knoxville, Tennessee \\
\hline Texas & $\mathrm{S}$ & University of Texas & Austin, Texas \\
\hline Texas A\&M & S & Texas A\&M University & College Station, Texas \\
\hline Texas Tech & S & Texas Tech University & Lubbock, Texas \\
\hline Toronto & $\mathrm{C}$ & University of Toronto & Toronto, Ontario \\
\hline Tulane & $\mathrm{P}$ & Tulane University & New Orleans, Louisiana \\
\hline Utah & S & University of Utah & Salt Lake City, Utah \\
\hline Vanderbilt & $\mathrm{P}$ & Vanderbilt University & Nashville, Tennessee \\
\hline Virginia & $\mathrm{S}$ & University of Virginia & Charlottesville, Virginia \\
\hline Virginia Tech & S & Virginia Polytechnic Institute \& State University & Blacksburg, Virginia \\
\hline Washington & S & University of Washington & Seattle, Washington \\
\hline Washington State & $\mathrm{S}$ & Washington State University & Pullman, Washington \\
\hline Washington U.-St. Louis & $\mathrm{P}$ & Washington University & St. Louis, Missouri \\
\hline Waterloo & $\mathrm{C}$ & University of Waterloo & Waterloo, Ontario \\
\hline Wayne State & S & Wayne State University & Detroit, Michigan \\
\hline Western Ontario & $\mathrm{C}$ & University of Western Ontario & London, Ontario \\
\hline Wisconsin & $\mathrm{S}$ & University of Wisconsin & Madison, Wisconsin \\
\hline Yale & $\mathrm{P}$ & Yale University & New Haven, Connecticut \\
\hline York & $\mathrm{C}$ & York University & North York, Ontario \\
\hline Boston Public Library & $\mathrm{N}$ & Boston Public Library & Boston, Massachusetts \\
\hline CISTI & $X$ & Canada Inst. for Scientific \& Technical Info. & Ottawa, Ontario \\
\hline Ctr. for Research Libs. & $\mathrm{N}$ & Center for Research Libraries & Chicago, Illinois \\
\hline Library of Congress & $\mathrm{N}$ & Library of Congress & Washington, DC \\
\hline Natl. Agricultural Lib. & $\mathrm{N}$ & National Agricultural Library & Beltsville, Maryland \\
\hline Library and Archives Canada & $X$ & Library and Archives Canada & Ottawa, Ontario \\
\hline Natl. Library of Medicine & $\mathrm{N}$ & National Library of Medicine & Bethesda, Maryland \\
\hline New York Public Library & $\mathrm{N}$ & New York Public Library & New York, New York \\
\hline New York State Library & $\mathrm{N}$ & New York State Library & Albany, New York \\
\hline Smithsonian Institution & $\mathrm{N}$ & $\begin{array}{l}\text { New York State Library } \\
\text { Smithsonian Institution }\end{array}$ & Washington, DC \\
\hline
\end{tabular}

